\title{
ENSEMBLES ET MORPHISMES STRATIFIÉS ${ }^{1}$
}

\section{R. THOM}

La catégorie des complexes simpliciaux, et des applications simpliciales, si elle permet une description explicite des types topologiques des espaces et des applications correspondantes, n'en présente pas moins deux inconvénients:

(1) Elle exige en général une subdivision trop fine, topologiquement arbitraire, de l'espace sous-jacent. (On sait par exemple le nombre élevé de sommets requis pour une triangulation d'un objet aussi simple que le tore $T^{2}$.)

(2) Certaines applications algébriques très simples, comme l'application du plan Ouv sur le plan Oxy définie par $x=u, y=u v(\sigma$ Prozess de Hopf) ne peuvent être réalisées simplicialement.

C'est dans le but de pallier ces défauts que nous introduisons ici la catégorie des ensembles et morphismes stratifiés. La présentation donnée ici est le fruit d'un long processus d'approximations successives, qui n'a sans doute pas atteint son état terminal. J'espère néanmoins que l'essentiel de la structure peut être considéré comme définitivement dégagé. Qu'il me soit permis de remercier ici tous ceux qui, dans divers séminaires, (à l'I.H.E.S. de Paris, à Brandeis) m'ont aidé de leurs conseils, de leurs critiques, ou de leurs rédactions. Je citerai en particulier: H. Cartan, D. Fotiadi, H. Hironaka, H. Levine, S. Lojasiewicz, J. Mather, B. Morin, F. Pham, M. H. Schwartz, O. Zariski. On reconnaitra au passage le rôle fondamental des idées de $\mathrm{H}$. Whitney, qui, en introduisant les propriétés (A) et (B) des ensembles analytiques, a fait faire à la théorie un progrès décisif.

\section{ThÉorie Topologique}

A. Une construction auxiliaire. Soit $X \stackrel{f}{\rightarrow} Y$ une application continue d'un espace topologique $X$ dans une espace $Y$. Munissons la réunion disjointe $X \cup Y$ de la topologie engendrée par les ouverts suivants:

(1) $U \subset X$, où $U$ parcourt l'ensemble des ouverts de $X$

(2) $f^{-1}(V) \cup V$, où $V$ parcourt l'ensemble des ouverts de $Y$.

Soit $M(f)$ l'espace ainsi défini; l'application $f$ se factorise en $X \stackrel{i}{\rightarrow} M(f) \stackrel{j}{\rightarrow} Y$, où $i$ est une injection ouverte, et $j$ une surjection.

Etant donné un diagramme commutatif d'applications de la forme

\footnotetext{
1 Research supported by NSF GN 530 to the American Mathematical Society.
} 


$$
\begin{gathered}
X \stackrel{f}{\rightarrow} Y \\
h \downarrow \quad k \downarrow \\
X^{\prime} \underset{f^{\prime}}{\rightarrow} Y^{\prime}
\end{gathered}
$$

le couple des applications $h, k$ permet de définir une application induite de $M(f)$ dans $M\left(f^{\prime}\right)$, ainsi qu'on le vérifie immédiatement. De même, à tout système d'espaces $X_{i}$ liés par des applications continues $X_{i} \rightarrow X_{j}$ formant un diagramme $D$ saturé pour la composition des flèches, la construction précédente généralisée associe un espace $M(D)$, et un morphisme d'un diagramme $D$ dans un diagramme $D^{\prime}$ induit fonctoriellement une application de l'espace $M(D)$ dans l'espace $M\left(D^{\prime}\right)$.

Même si $X$ et $Y$ sont des espaces séparés, l'espace $M(f)$ associé à une application continue $X \stackrel{f}{\rightarrow} Y$ est en général non séparé (le long de l'image fermée de $Y$ dans $M(f))$. Pour obvier à cette difficulté, on considérera, dans notre théorie, des morphismes locaux de $X$ dans $Y$ : on se donnera un filtre $X_{\alpha}$ d'ouverts de $X$, pour chaque $X_{\alpha}$ une application $f_{\alpha}$ de $X$ dans $Y$, avec la condition que si $X_{\beta} \subset X_{\alpha}, f_{\beta}$ est la restriction de $f_{\alpha}$ à $X_{\beta}$. Si dès lors, l'intersection des $X_{\alpha}$ est vide, alors l'espace $M(f)$ défini par les ouverts de la forme $U$ ( $U$ ouvert de $X$ ), et $f_{\alpha}^{-1}(V) \cup V, V$ ouvert de $Y$, est alors séparé si $X$ et $Y$ le sont.

Dans la théorie qui suit, tous les espaces stratifiés qu'on va définir seront séparés. C'est là probablement une restriction excessive, car de nombreux espaces intéressants, comme certains quotients d'actions de groupe, ou de feuilletages, ont souvent une sorte de structure stratifiée, et sont cependant non séparés. Ici encore, une généralisation de la théorie serait souhaitable.

B. Notion de submersion contrôlée. Soient $X$ et $Y$ deux variétés différentiables paracompactes (séparées, réunions dénombrables de compacts); si une application $f$ de $X$ sur $Y$ est de rang maximum (égal à la dimension de $Y$ ) et si $f$ est propre, alors $f$ est une fibration; ce résultat classique est vrai également si $X$ est une variété à bord, pourvu que la restriction de $f$ au bord $\partial X$ de $X$ soit une submersion (application partout de rang maximum). On appellera surmersion une submersion surjective.

Lorsque l'application submersive $f$ n'est plus propre, on ne peut rien dire en général. Nous dirons que la submersion $f \operatorname{de} X$ sur $Y$ est une submersion contrôlée, s'il existe une fonction positive réelle $C^{\infty} \mathrm{g}: X \rightarrow R$, la fonction de contrôle, ayant les propriétés suivantes:

(i) $g$ est strictement positive, et il existe un $a>0$, tel que la différ- 
entielle $d g$ n'est nulle en aucun point de la contre-image $\left.g^{-1}(] 0 a\right]$ ).

(ii) $f$ restreinte à la variété à bord $g \geqq \epsilon$ est une submersion propre sur $Y$ pour tout $\epsilon$ tel que $(0<\epsilon<a)$.

THÉoR̀̀me 1. B.1. Toute surmersion contrôlée $X \stackrel{f}{\rightarrow} Y$ est une fibration différentiable.

DÉmonstration. On munit $X$ d'une métrique riemannienne complète (par le théorème de Hopf-Rhinow); dans toute fibre de la forme $f^{-1}(y)$, on forme le gradient de la fonction de contrôle $g$, grad $g$. En intégrant ce champ de vecteurs, on peut étendre une trivialisation locale de $f \mid g \geqq \epsilon \rightarrow Y$ à $f \mid g \geqq \epsilon^{\prime}, \epsilon^{\prime}<\epsilon$, et par suite à tout $X$.

La démonstration précédente ne fait intervenir que la propreté locale (au-dessus d'un voisinage d'un point $y \in Y$ ) de la restriction $f \mid g \geqq \epsilon$. Ceci justifie la définition:

DÉfinition 1.B.1. Soit $X \stackrel{f}{\rightarrow} Y$ une submersion, et $g$ une fonction de $X$, différentiable, strictement positive, telle que $d g$ ne s'annule en aucun point de $\left.\left.g^{-1}\right] 0, a\right]$. On dira que $g$ est une fonction de contrôle locale pour $f$, si à tout compact $K$ de $Y$, on peut associer un $\eta(K)<a$, positif, tel que la restriction $f \mid g \geqq \epsilon \cap f^{-1}(K) \rightarrow K$, soit une submersion propre pour tout $\epsilon<\eta(K)$.

Corollaire 1. B.2. Si la surmersion $f$ admet une fonction de contrôle locale $g$, $f$ est une fibration différentiable.

Définition 1.B.2. Tapis d'une variêté. On appelle tapis d'une variété non compacte $X$ une fonction de contrôle pour la surmersion triviale $f: X \rightarrow$ point.

Proposition 1.B.3. Pour qu'une variété $X$ admette un tapis, il faut et il suffit que $X$ soit difféomorphe à l'intérieur d'une variété compacte à bord $M$.

En effet, l'intégration du champ grad $g$ (pour une métrique riemannienne complète) permet de réaliser ce difféomorphisme entre $X$ et $g$. La condition nécessaire est évidente, car si $X$ est l'intérieur de $M$, la fonction distance au bord de $M$ (pour une métrique riemannienne sur $M$ ) est un tapis pour $X$.

On sait [1] qu'une variété $X$ est difféomorphe à l'intérieur d'une variété à bord compacte $M$, si elle est connexe, simplement connexe, simplement connexe à l'infini, et si son homologie entière est de type fini. L'existence d'un tapis $\tau_{X}$ pour une variété $X$ n'est donc pas une grave restriction.

Proposition 1.B.4. Soit $X \stackrel{f}{\rightarrow} Y$ une surmersion, et $g$ une fonction 
de contrôle locale pour $f$; si h est un tapis de $Y$, il existe une fonction de contrôle globale $g_{1}$ pour $f$, où $g_{1}$ est, au voisinage de l'infini, de la forme $g / s(h \circ f)$, où $s$ est une fonction monotone croissante, $C^{\infty}$.

DÉmonstration. Pour tout nombre positif $b$ assez petit, considérons le compact de $Y$ défini par $h \geqq b$. Il existe une valeur $u(b)>0$ telle que, $f \mid g \geqq \alpha \quad \alpha<u(b)$ soit propre, et cette fonction $u(b)$ est monotone croissante, avec, si l'on veut, $u(0)=0$. On pourra donc minorer cette fonction $u(b)$ par une fonction $C^{\infty}$ croissante, $u(b)>s(b)$, avec $s(0)=0$. La fonction $g_{1}=g / s(h \circ f)$ est alors une fonction de contrôle globale.

Fonctions de contrôles équivalentes. Soient deux fonctions $g, g_{1}$ strictement positives sur $X$, sans point singulier pour des valeurs assez petites. Supposons:

(i) que $g<a, g_{1}<b$, définissent dans $X$ le même filtre (à tout $a>0$ correspond un $b>0$ tel que $g_{1}<b$ est contenu dans $g<a$, et réciproquement).

(ii) la submersion de $f$ de $X$ sur $Y$ est de rang maximum sur les variétés de niveau $g=a, g_{1}=b$, pour tout $a, b$ assez petit. Alors, si $g$ est une fonction de contrôle locale ou globale pour $f$, il en va de même pour $g_{1}$, et inversement. Les fonctions de contrôles $g$ et $g_{1}$ seront dites alors équivalentes.

DÉfinition 1.B.3. Espace stratifié défini par une submersion contrôlée. Soient $X \stackrel{\rho}{\rightarrow} Y$ une submersion contrôlée par $g$. On appellera espace stratifié engendré par $f$ l'espace $M(f)$ obtenu en prenant sur la réunion disjointe $X \cup Y$ la topologie définie par:

(i) tous les ouverts $U$ de $X$,

(ii) tous les ensembles de la forme $\left\{(g<\epsilon) \cap f^{-1}(V)\right\} \cup V$, où $V$ parcourt une base des ouverts de $Y$.

L'espace ainsi obtenu est séparé; si on remplace le contrôle $g$ par un contrôle $g_{1}$ équivalent, on ne change pas l'espace $M(f)$. Car, pour tout voisinage $V$ relativement compact, les ouverts $\left\{(g<a) \cap f^{-1}(V)\right\}$ $\cup V$ et $\left\{\left(g_{1}<b\right) \cap f^{-1}(V)\right\} \cup V$ définissent le même système fondamental de voisinages.

Définition 1.B.4. Deux surmersions contrôlées de $X$ sur $Y, f_{0} f_{1}$ seront dites homotopes, s'il existe une surmersion contrôlée $F: X \times I$ $\rightarrow Y \times I$ dont les restrictions aux faces $t=0, t=1$ sont $f_{0}, f_{1}$ (les contrôles de $f_{0}, f_{1}$ étant les restrictions du contrôle de $F$ ).

THÉOR̀ेme 1.B.5. Si $f_{0}, f_{1}$ sont deux surmersions contrôlées de $X$ sur $Y$, homotopes, alors les deux espaces stratifiés associés, $M\left(f_{0}\right), M\left(f_{1}\right)$ sont homéomorphes.

Démonstration. Soit $G$ la fonction de contrôle de l'homotopie; on 
relève le champ de vecteurs unitaire sur $I$ en un champ de vecteurs tangent aux variétés de niveau de $G$ dans $X \times I$; l'application $F$ étant propre sur $\{G=a\} \cap F^{-1}(V) \rightarrow V$, on peut intégrer ce champ de vecteurs, ce qui permet de montrer que les sections $\left\{g_{0}<a\right\} \cap f_{0}^{-1}(V)$ sont transformées par ce champ en $\left\{g_{1}<a\right\} \cap f_{1}^{-1}(V)$; la topologie définissant $M\left(f_{0}\right)$ est ainsi transformée en la topologie définissant $M\left(f_{1}\right)$.

Composition de submersions contrôlées. Soient $X \stackrel{p}{\rightarrow} Y \stackrel{q}{\rightarrow} Z$ deux submersions contrôlées, resp. par $g: X \rightarrow R, h: Y \rightarrow R$ (localement). Si $K$ est un compact de $Z$, l'ensemble de $Y$ défini par $\{h \geqq a\} \cap q^{-1}(K)$ $a<\epsilon(K)$, est appliqué proprement sur $K$ par $q$, et est donc compact. Pour la même raison, l'ensemble $\{f \geqq b\} \cap p^{-1}\left(\{h \geqq a\} \cap q^{-1}(K)\right)$ est compact, si $b<\eta(K)$; si $s$ est une fonction $C^{\infty}$ croissante telle que $s(\eta(K))=\epsilon(K)$, on vérifie que la fonction $\operatorname{Inf}(h \circ p, s(g))$ est une fonction de contrôle locale pour $q \circ p$. Une telle fonction est une fonction différentiable à coins (i.e. une fonction qui s'écrit localement comme Inf $x_{i}$ où les $x_{i}$ sont des fonctions coordonnées locales). Il est clair qu'on pourrait obtenir un contrôle $C^{\infty}$ équivalent en lissant la fonction dans un voisinage tubulaire de la variété coin.

La même construction s'étend à une chaîne d'un nombre quelconque de submersions contrôlées: $X_{1} \rightarrow X_{2} \rightarrow \cdots \rightarrow X_{k}$.

C. Théorie générale des ensembles stratifiés. Un ensemble stratifié $E$ est un espace séparé qui se présente comme une réunion finie de variétés $C^{\infty}$ connexes, les strates $X_{i}$ de $E$. L'espace $E$ peut s'obtenir à partir de la réunion disjointe des $X_{i}$ par un système de relations d'équivalence, que nous allons décrire, et qui permettent de "recoller" les strates entre elles.

On va définir inductivement les ensembles stratifiés par récurrence sur le nombre de leurs strates.

Les ensembles stratifiés à une strate sont les variétés $C^{\infty}$ connexes compactes, ou paracompactes munies d'un tapis (donc, difféomorphes à l'intérieur d'une variété à bord compacte). Les ensembles stratifiés à deux strates $X, Y$ sont ainsi définis: On se donne un ouvert $U \subset X$ (le "lambeau d'incidence") et une surmersion $k: U \rightarrow Y$, admettant pour fonction de contrôle locale un tapis de $X$. Alors l'espace stratifié $M(k)$ associé à cette surmersion représente le mode général de définition d'un ensemble à deux strates $X, Y$ où $\operatorname{dim} Y<\operatorname{dim} X$. On représentera par le symbole $Y<X$ ( $Y$ incidente à $X)$ cette situation.

De manière générale, un ensemble stratifié à $(k+1)$ strates s'obtient en prenant un ensemble $E$ stratifié à $k$ strates, et une variété à bord $M$ de dimension $\geqq \operatorname{dim} E$. On envoie alors le bord $V$ de $M$ (ou une partie de ce bord) dans $E$ par une application de recollement $h$ astreinte à une condition énoncée ci-dessous (condition Att); l'espace 
quotient de la réunion disjointe $E \cup M$ par la relation d'équivalence définie par $h$ définit un espace à $(k+1)$ strates.

DÉFInition 1.C.1. Introduisons d'abord, formellement, le schéma $d$ 'incidence d'un ensemble stratifié. A toute strate $X$ de dimension $r$ se trouve associé un nombre fini de strates de dimension inférieure à $r$, $Y_{1} \ldots Y_{s}$, les strates du bord de $X$ (ou incidentes à $X$ ). On note $Y_{i}<X$ cette situation. La relation $<$ est transitive pour les ensembles stratifiés compacts. On appelera étoile de $Y$ (noté St $Y$ ) l'ensemble des strates $X_{i}$ telles que $Y<X_{i}$; une suite de strates $X_{j}$ telles que $X_{1}<X_{2}$ $<\cdots<X_{k}$ sera appelée une chaîne de strates de longueur $k$. A tout couple de strates telles que $Y<X$, se trouve associé un ouvert de $X$, $L_{Y}(X)$, le lambeau d'incidence de $X$ relatif à $Y$. Sur $L_{Y}(X)$ est définie une surmersion $k_{Y X}: L_{Y}(X) \rightarrow Y$. Sur ce système des $L$ et des $k$, on fait l'hypothèse suivante, de transitivité: Si $Z<Y<X$, alors $L_{Y}(X)$ $\cap k_{Y X}^{-1}\left(L_{Z}(Y)\right) \subset L_{Z}(X)$ et, dans cette intersection, on a: $k_{Z X}=k_{Z Y} \circ k_{Y X}$ (Trans).

Les submersions $k_{Y X}$ admettent également un système de fonctions de contrôle qu'on définira inductivement. On suppose aussi que les lambeaux d'incidence satisfont à la propriété de disjonction:

$$
\text { Si } L_{Y}(X) \cap L_{Z}(X) \neq \varnothing, \quad \text { alors } Y<Z \text { ou } Z<Y \text {. }
$$

Etant donnée une strate $X$ de l'ensemble stratifié $E$, on appellera "tube de $X$ ", noté $L X$, la réunion des lambeaux d'incidence $L_{X}(Y)$, où $Y$ parcourt l'étoile de $X$, plus $X$ elle-même. Cette réunion est supposée munie de la topologie induite de $E$; pour cette topologie, l'ensemble des rétractions $k_{X Y}: L_{X}(Y) \rightarrow X$ définit une rétraction continue de $L X$ sur $X$, notée $k_{X}$, dont la restriction á chaque lambeau $L_{X}(Y)$ est une surmersion.

Nous sommes maintenant en mesure de préciser la condition imposée à l'application $h: V \rightarrow E$ qui attache le bord $V=\partial M$ dans $E$. C'est:

DÉFinition 1.C.2. Pour toute strate $X \subset E$, la composée:

$$
k_{X} \circ h: h^{-1}(L X) \rightarrow X
$$

est une surmersion, à moins que $h^{-1}(L(X))$ ne soit vide. Soit dès lors $A$ l'espace quotient obtenu de la réunion disjointe $E \cup M$ par l'identification $h: V \rightarrow E$. Il est aisé de définir dans $A$ les strates, les lambeaux et les applications $k$; on prend d'abord un voisinage tubulaire du bord $V$ dans $M$, soit $s: V \times I \rightarrow V$ une rétraction de ce voisinage sur le bord; désignons par $U$ l'intérieur $M-V$ de $M ; U$ devient une nouvelle strate de $A$, et il reste à définir le bord de $U$ dans $A$, les lambeaux d'incidence relatifs à $U$ et leurs rétractions. Une strate $X$ de $E$ est 
incidente à $U$ si $h^{-1}(L X)$ est non vide. (Comme l'image $h(V)$ est compacte, elle contient l'adhérence $\bar{X}$ de toute strate $X$, dès qu'elle contient un point de $X$.) Or, on démontre inductivement que la frontière topologique d'une strate $X$ est constituée de l'ensemble des strates incidentes à $X$, (et celles-là seulement). On prend alors pour lambeau $L_{X}(U)$ l'image (après identification sur $E \cup M$ ), de l'ouvert $s^{-1}\left(h^{-1}(L X)\right)$. Il est aisé de vérifier que la condition (Dis) est alors satisfaite; la rétraction $k_{X U}$ est définie-avant identification$\operatorname{par} k_{X} \circ h \circ s$. La relation (Trans) est alors de vérification immédiate. Il est de plus clair que l'adhérence topologique de $U$ dans $A$ consiste en l'ensemble des strates du bord: en effet, dès qu'un point d'une strate $X$ de $E$ est contenu dans $h(V), X$ est tout entière contenue dans $h(V)$ (à cause de la condition (Att)).

I. D. Normalisation des applications d'attachement et des lambeaux d'incidence. On va montrer qu'on peut-sans changer la topologie de l'espace $A$-remplacer l'application d'attachement $h: V \rightarrow E$ par une application $h^{\prime}$ normalisée de $h$, telle que la restriction de $h^{\prime}$ à la contreimage d'une strate $X$ de $E$ soit une fibration, dont la fibre est une variété à coins de dimension maximum dans $V$.

Dans tout ce qui suit, nous supposerons dorénavant l'espace stratifié compact; il suffit pour cela de postuler que toutes les applications d'attachement $h: V \rightarrow E$ sont définies sur tout $V$.

Définition 1.D.1. Présentation d'un ensemble stratifié. Soit $E$ un ensemble stratifié de strates $X_{i}$; une présentation de $E$ consiste dans la donnée, pour chaque strate $X_{i}$ d'une réunion de variétés à coins $X_{i}=\bigcup_{\omega \in \Omega\left(X_{i}\right)} M_{i}^{\omega}$ fermées, indexées par l'ensemble $\Omega\left(X_{i}\right)$ des chaînes $\omega$ de strates dont le dernier terme est $X_{i}$; la chaine triviale $\left\{X_{i}\right\}$ réduite à $X_{i}$ seul définit une variété de la forme $\tau_{X_{i}} \geqq a$, dont l'intérieur est difféomorphe à $X_{i}$. Le bord de chaque $M_{i}^{\omega}$ est une réunion de variétés à coins $M_{i}^{\omega R}$ qu'on peut indexer de la manière suivante: dans toute chaîne $\omega$, marquons un certain sous-ensemble $K$ de strates (sauf $X_{i}$ elle-même). Si on efface ces sous-ensembles, on obtient encore une chaîne de l'ensemble $\Omega\left(X_{i}\right)$, soit $\omega^{\prime}=\omega-K$. On désignera par $\omega_{K}$ la chaîne $\omega$ où l'on a marque par l'accent comme devant être supprimé-le sous-ensemble $K$ de strates. Tout symbole $\omega_{K}$ définit alors une variété $M_{\omega_{K}} \in \partial M_{\omega} \cap \partial M_{\omega-K}$ et l'on a alors pour définir les intersections des variétés $M_{\omega_{R}}$ les règles suivantes:

$$
\begin{aligned}
& M_{\omega} \cap M_{\omega-K}=M_{\omega_{K}}, \\
& M_{\omega_{J}} \cap M_{\omega_{K}}=M_{\omega_{J \cup K}} .
\end{aligned}
$$

On verra plus tard que, si $|J|$ désigne le nombre de strates de l'en- 
semble $J, M_{\omega_{J}}$ est de codimension $|J|$ dans $X_{i}$. Si les ensembles $J$ et $K$ sont disjoints, $M_{\omega_{J}}$ coupe $M_{\omega_{K}}$ transversalement le long de $M_{\omega_{J} \cup_{K}}$. Deux variétés $M_{\omega_{1}}, M_{\omega_{2}}$ ne se rencontrent que si $\omega_{1}$ et $\omega_{2}$ sont des souschaînes d'une même chaîne $\omega \in \Omega\left(X_{i}\right)$.

Soient dès lors $X, Y$ deux strates telles que $Y<X$. Désignons par $O(X, Y)$ la totalité des chaînes de strates aboutissant à $X$ et contenant $Y$; la réunion $U M_{\omega}, \omega \in O(X, Y)$ constitue le "lambeau d'incidence normalisê" $L_{Y}(X)$. La rétraction $k_{Y X}: L_{Y}(X) \rightarrow Y$ est une fibration, telle que l'image $k_{Y X}: M_{\omega}$ soit la variété $M_{\omega^{\prime}}$, où $\omega^{\prime}$ est la chaîne $\omega$ arrêtée à la strate $Y$. Il reste à préciser la topologie de ces variétés $M_{\omega}$, ainsi que celle définie sur leur réunion par les applications d'attachement $k$ dans l'espace quotient $E$. Introduisons dans ce but une notion:

DÉfinition 1.D.2. Secteur associê à une châ̂ne de morphismes. Considérons, dans l'espace euclidien $R^{k}$ de coordonnées $x_{i}, i=1,2$, . . , $k$, le simplexe standard de dimension $k \Delta^{k}$ défini par les inégalités: $x_{2} \leqq x_{1}, x_{3} \leqq x_{2} \cdots x_{k} \leqq x_{k-1}$ contenu dans le cube unité $0 \leqq x_{i} \leqq 1$. On désignera par $a_{0}\left(x_{i}=0\right), a_{1}\left(x_{1}=1, x_{i}=0, i>1\right), \cdots$, $a_{j}\left(x_{1}=x_{2}=\cdots=x_{j}=1, x_{j+8}=0\right)$ ses sommets successifs.

Soit maintenant $A_{0} \rightarrow A_{1} \rightarrow \cdots \rightarrow A_{i} \rightarrow \cdots \rightarrow A_{k}$ une suite d'applications continues entre $(k+1)$ espaces topologiques. On considère l'espace fonctionnel $S$ des applications $F$ de sommets $a_{i}$ du simplexe $\Delta^{k}$ dans la somme disjointe $A_{0} \cup \ldots \cup A_{k}$ assujetties aux conditions suivantes:

$$
F\left(a_{0}\right) \in A_{0} \cdots F\left(a_{i}\right) \in A_{i} \cdots F\left(a_{k}\right) \in A_{k}
$$

avec $f_{i} F\left(a_{i}\right)=F\left(a_{i+1}\right)$.

Ces conditions définissent un sous ensemble fermé du joint des espaces $A_{0} * \cdots * A_{k}$, qu'on désignera par le symbole $S_{\omega}, \omega$ désignant la chaîne $A_{0} \rightarrow \cdots \rightarrow A_{i} \rightarrow A_{k}$ et qu'on appelle le secteur associé à la chaîne $\omega$.

Le secteur associé à une chaîne à deux éléments, un morphisme $f: A_{0} \rightarrow A_{1}$, n'est autre que le "mapping cylinder" de l'application $f$. On observera que le secteur associé à une chaîne $\omega^{\prime}$ de morphismes contient canoniquement le secteur associé à toute sous-chaîne $\omega$ $=\omega^{\prime}-K$ de $\omega^{\prime}$ (où, si l'on supprime un espace $A_{i}$, on compose les applications adjacentes: $A_{i-1} \stackrel{f_{i-1}}{\longrightarrow} A_{i} \stackrel{f_{i}}{\rightarrow} A_{i+1}$ devenant $\left.A_{i-1} \stackrel{f_{i} f_{i-1}}{\longrightarrow} A_{i+1}\right)$. Il suffit pour cela de se restreindre à la face du simplexe $\Delta^{K}$ définie par les sommets figurant dans $\omega^{\prime}$. On désignera par $S_{\left[\omega^{\prime}, K\right]}$ ce soussecteur.

Revenons maintenant à l'espace stratifié $E$, et considérons une chaîne de strates $X_{0}<X_{1}<\cdots<X_{k}$; dans chacune de ces strates $X_{i}$, on a défini une variété à coins associée à la sous-suite tronquée 
$X_{0}<X_{1}<\cdots<X_{i}$, soit $M_{\omega} i$; cette variété admet également dans son bord une sous-variété définie par le symbole $\hat{X}_{0}<\cdots<\hat{X}_{i-1}$ $<\hat{X}_{i}$, soit $N_{\omega} i$; il existe alors une suite canonique de morphismes surjectifs (restrictions des $k_{X_{i-1} X_{i}} \grave{a} N_{i}$ )

$$
X_{0} \stackrel{\hat{k}_{01}}{\longleftarrow} N_{1} \stackrel{\hat{k}_{12}}{\longleftarrow} N_{2} \ldots \stackrel{\hat{k}_{K-1 K}}{\longleftarrow} N_{K} .
$$

La réunion des variétés $M_{\omega} i$, munie de la topologie quotient définie par les applications d'attachement $k_{X_{i-1} X_{i}}$ n'est autre que le secteur associé à la chaîne de morphismes $(\omega)$.

Ainsi, pour un espace stratifié à deux strates $X, Y, Y<X$, l'image par le recollement $k_{Y X}$ du lambeau d'incidence $L_{Y}(X)$ avec $Y$ n'est autre que le mapping cylinder de l'application surjective

$$
\hat{k}:[\hat{Y}, X] \rightarrow Y \text { où }[Y, X] \text { désigne } M_{[Y, X]} .
$$

L'identification du quotient de la réunion des $M_{\omega^{i}}$, $\omega^{i}$ suites tronquées de $\omega$, avec le secteur $S_{\omega}$ est compatible avec les injections $M_{\omega_{R}} \rightarrow M_{\omega}$ décrites plus haut.

Soient $\omega$ une chaîne de strates, $\omega^{\prime}$ une sur-chaine de $\omega$ et $K=\omega^{\prime}-\omega$, l'ensemble différence. Les injections canoniques $M_{\omega_{K}} \rightarrow M_{\omega}$, effectuées pour toutes les chaînes tronquées de $\omega^{\prime}$ dans les $M_{\omega^{\prime}}$ passent au quotient et permettent d'injecter un sous-secteur $S_{\omega^{\prime}, K}$ de $S_{\omega^{\prime}}$ dans $S_{\omega^{\prime}}$.

Ces définitions étant posées, on va maintenant montrer le

THÉORÈmE 1.D.1. Tout espace siratifié compact admet une présentation associée auX applications $k_{Y X}$ données.

La démonstration se fait par récurrence sur le nombre des strates; la propriété est, on l'a vu, vraie d'un ensemble stratifié compact à deux strates; en effet, si $Y<X, Y$ compacte, $X$ est somme de deux variétés à bord: une variété $[X]$ définie par $\tau_{X} \geqq \epsilon, \tau_{X}$ tapis de $X$, et un lambeau d'incidence $L_{Y}(X)$, défini par $\tau_{X} \leqq \epsilon$; ce lambeau est la variété $M[Y, X]$ de la présentation; la variété $N=M[\hat{Y}, X]$ est le bord commun à $[X]$ et $M_{[Y, X]}$ défini par $\tau_{X}=\epsilon$.

Dans ce qui suit, on écrira pour simplifier $[\omega]$ au lieu de $M_{(\omega)}$. On va donc admettre que l'ensemble stratifié $E$, compact, à $(k-1)$ strates, est pourvu d'une présentation par des variétés à coins $[\omega]$, et des applications $k_{Y X}: L_{Y}(X) \rightarrow Y$. On forme un espace stratifié $A$ en attachant à $E$ une variété à bord $P$, dont le bord $V$ est envoyé dans $E$ par une application $g$ satisfaisant à la condition (Att) du 1.C. On va user d'une induction nouvelle relative à la "cohauteur" d'une strate de $E$ par rapport à $P$.

DÉfinition 1.D.3. Une strate $X$ de $E$ est dite de cohauteur $s$ relative à $P$, si $s+1$ est la plus grande longueur d'une chalne de strates 
commençant en $X$ et finissant en $P$. Ainsi, une strate $Y$ directement incidente à $P$ (sans strate intermédiaire $Z$ telle que $Y<Z<P$ ) est de cohauteur un.

Sur une strate $Y$ de cohauteur un par rapport à $P$, l'application d'attachement $g$, qui est submersive, sert à définir $k_{Y P}$. Toute variété de la présentation du type $[\omega, Y]$ s'étend en une variété de symbole $[\omega Y P]$, en prenant la contre-image par $g$ dans $V \times I$; sur $V \times 1$, on a la variété $[\omega \hat{Y P}]$; par passage au quotient sur la réunion $[\omega Y]$ $\cup[\omega Y P]$ définie par $k_{Y X}$, on a la topologie d'un secteur $S(\omega, Y, P)$ associé à $\hat{k}_{Y X}[\hat{\omega} \hat{Y} P] \rightarrow[\hat{\omega} Y] \rightarrow \cdots$. Considérons maintenant le cas d'une strate $Y$ de cohauteur 2.

On forme dès lors le tube $L(Y)$, réunion des lambeaux d'incidence $\bigcup_{X_{i}} L_{Y}\left(X_{i}\right)$, où $X_{i} \in \mathrm{St} Y$ dans $E$. On définira dans ce tube $L_{Y}$ une fonction $t$, nulle sur $Y$, égale à un sur le bord de $L_{Y}(Y)$. Dans chaque lambeau $L_{Y}\left(X_{i}\right)$, cette fonction $t$ est celle-là même qui apparait lorsqu'on identifie par quotient la réunion $L_{Y}\left(X_{i}\right) \cup Y$ au secteur $S\left(Y, X_{i}\right)$ (ici le "mapping cylinder" de $\left.k_{Y X_{i}}: \partial L_{Y}\left(X_{i}\right) \rightarrow Y\right)$. Par suite, cette fonction $t$ est de rang maximum dans chaque lambeau $L_{Y}\left(X_{i}\right)$; il en ira par suite de même dans toute contre-image $N\left(X_{i}\right)=g^{-1}\left(L_{Y}\left(X_{i}\right)\right)$ pour la fonction induite $t \circ g, g$ étant submersive sur $X_{i}$.

Introduisons maintenant le voisinage tubulaire $V \times I$, paramétré par $u$, de $V$ dans $P$; définissons, comme précédemment $g$ comme la composée $V \times I \rightarrow V \stackrel{g}{\rightarrow} E$. On voit que $N=\boldsymbol{g}^{-1}\left(L_{Y}\left(X_{i}\right)\right)$ a une structure de produit local de la forme ]0, 1] $\times] 0,1] \times F \times Y$ où $F$ est la fibre $\left(k_{Y X_{i}} \circ g\right)^{-1}(y) \cap \partial\{t \circ g=1\}$, car l'application définie par $\left(t, u, k_{Y X_{i}} \circ g\right)$ de $N$ sur le carré demi-ouvert $0<u \leqq 1,0<t \leqq 1$ multiplié par $Y$, est une submersion propre.

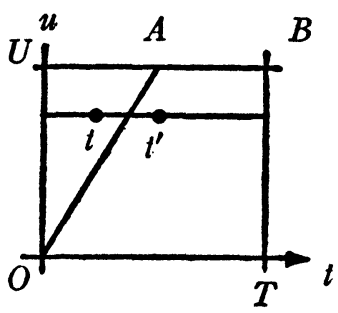

Figure 1

Dans ce carré, $O U B T, 0 \leqq u \leqq 1,0 \leqq t \leqq 1$, on va considérer la droite $O A$ d'équation $u=2 t$, qui rencontre la droite $U B(u=1)$ au point $A$ de coordonnées $t=\frac{1}{2}, u=1$. L'application $\Phi$ de $O U B T$ dans lui-même $(t, u) \stackrel{\Phi}{\rightarrow}\left(t^{\prime}, u^{\prime}\right)$ définie par $u^{\prime}=u 1-t^{\prime}=(1-u / 2)(1-t)$ envoie linéairement le carré $O U B T$ sur le trapèze $O A B T$ (Figure 1 ).

Normaliser l'application d'attachement \&, c'est remplacer, dans 
toute fibre $\left(k_{Y X_{i}} \circ \mathcal{g}\right)^{-1}(y), y \in Y$, l'application $\boldsymbol{g}$ par l'application $G$ ainsi définie:

$$
\begin{array}{rlll}
G\left(u, t^{\prime}, f\right) & =g(u, t, f) & \text { si } & t^{\prime}>u / 2, \quad f \in F \\
& =g(u, 0, f) \quad \text { si } \quad t^{\prime}<u / 2
\end{array}
$$

où $t^{\prime}$ et $t$ sont, dans le premier cas, liés par la relation $\Phi$. Dans l'application normalisée $G$, la contre-image $G^{-1}(Y)$ est une variété à bord, dont le bord est constitué de produits de la forme $O A \times F \times Y$; on globalise en effet l'application $G$ à tout $(V \times I) \cap \boldsymbol{g}^{-1}(L Y)$ en posant $G=\boldsymbol{g}$ en tout point $\operatorname{de} \boldsymbol{g}^{-1}(Y)$. Intuitivement, la normalisation a pour effet de remplacer la contre-image $g^{-1}(Y)$, qui peut être très irrégulière, par un épaississement (thickening) qui est une variété à bord lisse.

Vérifions que le remplacement de $\boldsymbol{\xi}$ par $G$ ne modifie pas la topologie de l'espace $A$ obtenu par recollement. La chose est évidente pour les points intérieurs à $P$; en un point de $X_{i}$, situé dans $k_{Y X_{i}}^{-1}(y)$ de parametre $t_{0}$, on peut décrire un système fondamental de voisinages dans $g^{-1} k_{\bar{Y} X_{i}}^{-1}(y)$, dans les deux cas, par les inégalités:

$$
\begin{array}{cc}
\text { Topologie }(g) & \text { Topologie }(G) \\
\left|t-t_{0}\right|<\epsilon & \left|G^{*}(t)-G^{*}\left(t_{0}\right)\right|<\epsilon \\
u<\epsilon_{1} & u<\epsilon_{1}
\end{array}
$$

Comme $G^{*}(t)=t^{\prime}=1+(t-1)(1-u / 2)=t-(u / 2) t+u / 2$ on voit de suite que ces systèmes de voisinages sont équivalents dans le carré demi-ouvert $O U B T$, et par suite aussi dans l'espace $N\left(X_{i}\right)$.

Pour un point $y \in Y$, un système fondamental de voisinages est défini par:

$$
\begin{aligned}
& \left\{\begin{array}{l}
g^{*} t<a \\
u<b
\end{array} \text { dans } g^{-1}\left(L_{Y}\left(X_{i}\right)\right) \quad \text { pour la topologie }(g)\right. \\
& \left\{\begin{array}{l}
G^{*} t<a^{\prime} \\
u<b
\end{array} \text { dans } G^{-1}\left(L_{Y}\left(X_{i}\right)\right) \quad \text { pour la topologie }(G)\right.
\end{aligned}
$$

Il est clair, comme $G^{*}(t)=t^{\prime}$ est fonction croissante de $u$, que le rectangle $b C D O$ est contenu dans un $G$-voisinage tel que le trapèze $b E d O$ (Figure 2). Par ailleurs, tout $g$-voisinage contient un $g$-voisinage où $b<2 a$, donc tel que le rectangle $b C^{\prime} A^{\prime} O$, lequel contient le $G$-voisinage $b C^{\prime} D^{\prime} O$ (Figure 3).

Pour un point $z \in Z$ d'une strate $Z$ du bord de $Y$, la démonstration précédente s'applique avec l'addendum suivant: un système fondamental de voisinages de $z$ exige en plus, pour être décrit, des inégali- 


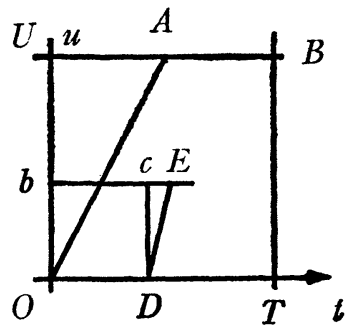

Figure 2

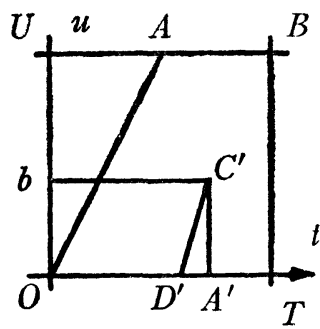

FIGURE 3

tés de la forme: $\left(k_{Z X} \circ g\right)^{*} \tau_{Z_{i}}<\epsilon$, où les $Z_{i}$ sont des strates de l'étoile de $Z$ incidentes à $Y$; or la normalisation n'affecte pas les applications $k_{Z X} \circ g$, qui sont égales à $k_{Z X} \circ G$; en effet, $g=G$ en dehors de la contreimage $g^{-1}\left(L_{Y}\right)$, et, dans cette contre-image, on a $k_{Z X} \circ g=k_{Z X} \circ G$; pour toute strate $Z$ telle que $Z<Y$, la normalisation laisse invariante les fibres $k_{Y X}^{-1}(y)$, et par suite aussi les fibres, plus grandes, de $k_{Z X} \circ g$ dans $g^{-1}\left(L_{Z}(X) \cap L_{Y}(X)\right), X \in \operatorname{Star} Y$, (en raison de (Trans)).

Après normalisation de l'application d'attachement $\xi$ en $G$, on peut étendre la présentation de $E$ en une présentation de $A$. Remarquons d'abord que $G$, telle que nous l'avons définie, n'est pas $C^{\infty}$ sur $G^{-1}\left(X_{i}\right)$; on devra donc la lisser au voisinage de la "face" $g^{*} t=1$, ce qui ne soulève aucune difficulté de principe.

On étendra la présentation de $E$ à $A$ par la règle suivante: si $M$ est une variété de présentation de symbole $\left[X_{i}\right]$ ou $\left[Y X_{i}\right]$ les variétés de symboles $[X P]$ ou $[Y X P]$ sont les contre-images par $G$ dans $V \times$ $\cap P:[Y X P]=G^{-1}[Y X]$ par exemple. La variété de symbole $[Y P]$ est également la contre-image $G^{-1}(Y)$ dans $V \times I \cap P$. Cette variété à bord a pour bords, conformément au schéma général, les variétés $\left[Y \hat{X}_{i} P\right]$ comme "surface latérale" et la variété $[\hat{Y} P]$ comme base. Voir Figure 4, pour une présentation d'un angle plan.

On peut définir alors sur la réunion de ces variétés, par passage au quotient, une structure de secteur. Pour définir l'application $k_{X P}$ on garde l'application $g$ initiale; pour définir $k_{Y P}$, on pose $k_{Y P}=G$ sur $[Y P]$, et $k_{Y P}=k_{Y X} \circ k_{X P}$ sur le "secteur triangulaire" [YXP].

Rappelons à ce propos que, dans une présentation, la rétraction $k_{X}$ du tube $L_{X}$ sur une strate $X$ est une rétraction par déformation dont les trajectoires forment un champ radial continu qui laisse invariantes toutes les strates de l'étoile de $X$, ainsi que les strates de la présentation de symboles $[X \omega]$. De plus, ce champ commute aux applications d'attachement $k_{Z Y}$, où $X<Z<Y$.

Nous pouvons maintenant aborder le pas central de l'induction; à supposer qu'on ait normalisé l'application d'attachement $g: V \rightarrow E$ sur toutes les strates $X$ de cohauteur inférieure à $(s-1)$, montrons 


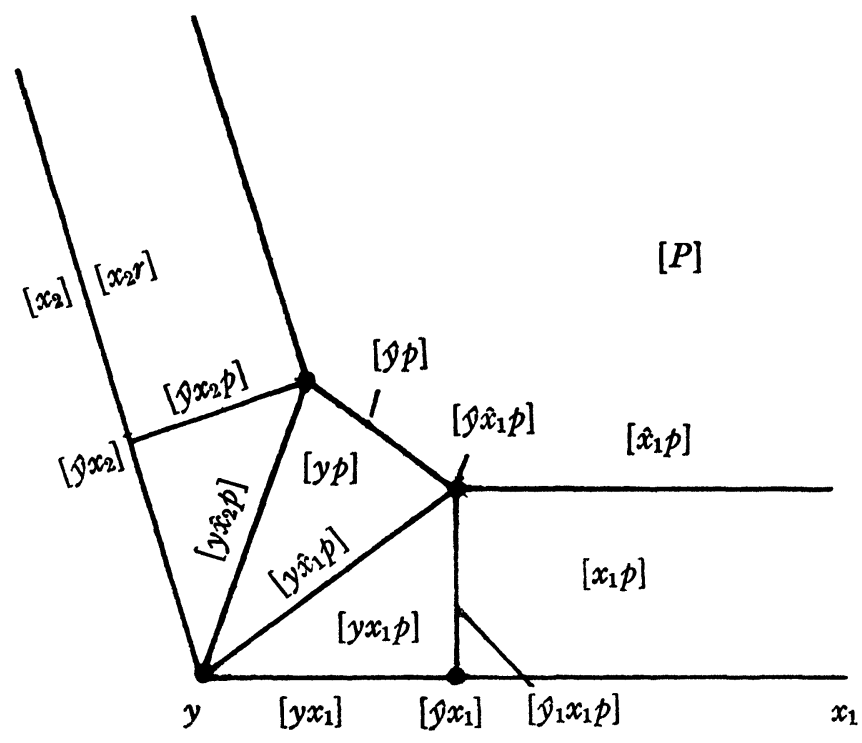

Figure 4

qu'on peut étendre cette normalisation à une strate de cohauteur's. Par section transversale par l'application $k_{X} \circ g$, on peut toujours se ramener au cas où la strate $X$ considérée est de dimension zéro; aussi l'appellerons nous 0 . Le tube $L_{0}$ a alors une structure de cône, et la rétraction $k_{0}: L_{0} \rightarrow 0$ est une rétraction par déformation dont les trajectoires définissent un champ $(r)$, différentiable dans chaque strate de la présentation et commutant aux $k$. On désignera par $t$ un paramétre radial pour cette transformation, valant zéro en 0 , et un sur le bord du tube $L_{0}$. (Ce paramètre $t$ est défini, bien entendu, par la structure en secteur des familles de variétés $\left[0 \omega_{i}\right]$ définies en tronquant une chaîne de strates d'origine 0 .)

Sur l'ensemble défini par $t=c s t e(0<t<1)$, l'application $g$ est normalisée puisqu'elle ne rencontre que des strates de cohauteur $s-1$. Il en résulte que la présentation donnée sur $L_{0}$ se relève en une présentation sur la contre-image $g^{-1}\left(L_{0}\right)$ dans $V \times I$ (de paramètre $u$ ); on relève alors le champ radial $(r)$ sur $L_{0}$ en un champ différentiable par morceaux qui laisse invariantes les variétés de la présentation induite sur $V \times I$. L'existence d'un tel relèvement $r^{\prime}$, tel que $g\left(r^{\prime}\right)=r$, s'établit par induction sur la cohauteur des strates de $L_{0}$. Pour une strate de cohauteur un, soit $X$, on relève le champ sur $X$, ainsi que sur toutes les strates de la forme $[Y \omega X]$ dans $V \times I$. C'est possible puisque $g$ est sur cette variété une fibration propre. Ce faisant le champ $r$ sera défini sur le bord des variétés de la forme $[Y P]$, où $Y$ est de cohauteur 
2. En se restreignant à la contre-image par $g$ d'une trajectoire de $r^{\prime}$ dans $Y$, on étendra $r$ dans [YP] par la propriété de prolongement classique des variétés à coins: si une fonction continue sur les faces d'un coin est differentiable dans toute face du coin (de toute dimension), elle provient d'une fonction différentiable de l'espace ambiant. Ainsi, on pourra de proche en proche définir le champ $r^{\prime}$ relevé du champ $r$. Le champ $r^{\prime}$ ainsi obtenu n'est que continu dans $V \times I$, différentiable par morceaux. Néanmoins, on peut l'intégrer isolément dans chaque strate de la présentation ce qui permet de définir une structure globale de produit au-dessus de $L_{0}-0$. On pourra dès lors, définir sur $N=\mathcal{B}^{-1}\left(L_{0}-0\right)$ une structure de produit de la forme ] 01 1] $\times$ ] 01 1] $\times F$, définie par la fibration $N \rightarrow I^{2}$ définie par $(u, t)$. On normalisera dès lors comme dans le cas de la cohauteur 2 , à l'aide d'une rétraction linéaire $\Phi$ définie dans le carré $0<u \leqq 1,0<t \leqq 1$.

Ainsi la contre-image de 0 par l'application $G$ normalisée de $g$ sera difféomorphe au produit de $F$ par une demi-droite. C'est donc, ici encore, une variété à bord, dont le bord est une réunion de variétés à coins. L'"ordre des coins" (i.e. la codimension maximum nécessaire pour définir le coin) ne peut excèder $s-1$.

L'extension de la présentation à $P$ se fera comme dans le cas de cohauteur 2: on posera $[O P]=G^{-1} 0$, mais, pour toutes les autres strates $X$ de $L_{0}$, on conserve $\boldsymbol{g}:[X P]=\boldsymbol{g}^{-1}(X)$. La vérification des propriétés sectorielles ne présente d'autre difficulté que celle de la longueur.

La description de la topologie d'un ensemble stratifié compact à l'aide de secteurs peut alors se faire par la procédure suivante: On construit d'abord le schéma d'incidence global entre strates $X_{i}$ de l'ensemble (on écrira pour la commodité $X \cdot \rightarrow Y$ au lieu de $Y<X$; rappelons que ce systéme de flèches est saturé pour la composition: si $X \cdot \rightarrow Y$ et $Y \cdot \rightarrow Z$, alors le schéma contient $X \cdot \rightarrow Z$ ). A toute flèche $X \cdot \rightarrow Y$ on associe une variêté d'incidence, qui est le bord de la variété de symbole $[Y X]$, soit $[\hat{Y}, X] ;$ c'est une variété à bords à coins fibrée sur $Y$; on se donne alors pour le système des variétés $[\hat{Y} X]$, leurs relations d'incidence: si $X \cdot \rightarrow Z \cdot \rightarrow Y$, on a une injection de $[\hat{Y} \hat{Z} X]$ dans le bord de $[\hat{Y} X]$ comme sous-variétés à coins, ... et ainsi de suite pour les variétés associées à une chaîne $X \rightarrow Z_{k} \rightarrow Z_{K-1} \rightarrow Z_{1} \rightarrow Y$. On se donne ensuite les applications $k_{Y X}$. Alors à toutes chaîne de strates correspond une chaine de morphismes $\hat{k}_{X}$ :

$$
\begin{aligned}
{\left[\hat{Y} \hat{Z}_{1} \hat{Z}_{2} \cdots \hat{Z}_{K} X\right] } & \stackrel{k_{Z_{k} X}}{\longrightarrow}\left[\hat{Y} \hat{Z}_{1} \hat{Z}_{2} \cdots Z_{K}\right] \cdot \rightarrow \cdots \\
& \longrightarrow\left[\hat{Y} \hat{Z}_{1} Z_{2}\right] \stackrel{k_{Z_{2} Z_{1}}}{\longrightarrow}\left[\hat{Y} Z_{1}\right] \stackrel{k_{Y Z_{1}}}{\longrightarrow}[Y] .
\end{aligned}
$$


On forme alors le secteur $S\left(Y Z_{1} Z_{2} \cdots Z_{K} X\right)$ associé à cette chaîne de morphismes. On opère sur la réunion de tous ces secteurs (pour toutes les chaînes) les identifications induites des injections [ $Y \hat{Z} X]$ $\rightarrow[Y X]$. L'espace quotient ainsi obtenu est l'espace stratifié $E$.

On pourrait démontrer directement par induction, à partir de la présentation en secteurs, que la projection $k_{Y}$ du tube $L_{Y}$ d'une strate $Y$ sur $Y$ est une fibration. Nous donnerons plus tard une démonstration de ce fait, comme cas particulier d'un théorème plus général.

1. E. Morphismes stratifiés. La théorie des morphismes stratifiés ne se présente de manière (relativement) claire que pour les ensembles stratifiés compacts. Nous ferons dorénavant cette hypothèse, sauf mention explicite du contraire.

Une application stratifiée $f: E \rightarrow E^{\prime}$ se factorise en une surjection $E \rightarrow A$ suivie d'une injection $A \rightarrow E^{\prime}$.

Une injection stratifiée $j: A \rightarrow E^{\prime}$ est ainsi définie: L'image $j(X)$ d'une strate de $A$ est une strate $X^{\prime}$ de $E^{\prime}$, de même dimension, $j$ est un difféomorphisme de $X$ sur $X^{\prime} ; j$ commute aux applications $k$ de recollement: si $X$ et $Y$ sont deux strates de $A$ telles que $Y<X, Y^{\prime}<X^{\prime}$ leurs images $L_{Y}(X), L_{Y^{\prime}}\left(X^{\prime}\right)$ leurs lambeaux d'incidence, alors, sur

$$
L_{Y}(X) \cap j^{-1} L_{Y^{\prime}}\left(X^{\prime}\right), \quad \text { on a: } j \circ k_{Y X}=k_{Y^{\prime} X^{\prime}} \circ j .
$$

Enfin, si $\tau_{X}, \tau_{X^{\prime}}$ désignent des tapis de $X$ et $X^{\prime}, j^{-1}\left(\tau_{X^{\prime}}\right)$ est un tapis de $X$ équivalent au tapis donné pour $A$.

Les injections ont donc une structure pratiquement triviale, et seules les surjections sont intéressantes.

Une surjection stratifiée $f: E \rightarrow A$ possède les propriétés suivantes:

(1) L'image $f(X)$ d'une strate $X$ de $E$ est une strate $X^{\prime}$ de $A$, et l'application restriction $f \mid X$ est une surmersion.

(2) La surmersion $f: X \rightarrow X^{\prime}$ est localement contrôlée par une fonction $c(f)$, telle que $\operatorname{Inf}\left(c(f), f^{*} \tau_{X^{\prime}}\right)$ est un tapis de $X$ pour tout tapis $\tau_{X^{\prime}}$ de $X^{\prime}$.

(3) $f$ commute aux applications $k$ : si $X$ et $Y$ sont deux strates de $E$, telles que $Y<X$, et si $X^{\prime}, Y^{\prime}$ sont leurs images par $f, L_{Y}(X), L_{Y^{\prime}}\left(X^{\prime}\right)$ les lambeaux d'incidence correspondants, alors sur $L_{Y}(X) \cap f^{-1}\left(L_{Y^{\prime}}\left(X^{\prime}\right)\right)$ supposé nonvide, on $a$ :

$$
f_{Y} \circ k_{Y X}=k_{Y^{\prime} X^{\prime}} \circ f_{X}
$$

lorsque $X^{\prime}$ et $Y^{\prime}$ sont distinctes; si $X^{\prime}$ et $Y^{\prime}$ sont confondues, alors on a: $f_{Y} \circ k_{Y X}=f_{X}$ en désignant par $f_{X}$ la restriction de $f$ à la strate $X$.

Le composé de deux morphismes stratifiés est un morphisme stratifié. Ceci est immédiat pour les compositions: Injection o Injection, 
et Surjection o Injection. La seule difficulté consiste à vérifier la condition 2 pour Surjection o Surjection.

Soient $E \stackrel{f}{\rightarrow} E^{\prime} \stackrel{g}{\rightarrow} E^{\prime \prime}$ une suite de surjections, $X \stackrel{f}{\rightarrow} Y \stackrel{g}{\rightarrow} Z$ une suite de strates images par $f$ et $g$. Soient $\tau_{Y}, \tau_{Z}$ des tapis de $Y$ et $Z, c(f), c(g)$ les fonctions de contrôle de $f$ et $g$ respectivement. Par hypothèse, Inf $\left\{c(f), f^{*} \tau_{Y}\right\}$ est un tapis pour $X$, quel que soit le tapis $\tau_{Y}$ de $Y$; on peut donc prendre pour $\tau_{Y}$ l'expression $\operatorname{Inf}\left\{c(g), g^{*} \tau_{Z}\right\}$ donnée par la surjection $g$. Ceci donne:

$$
\begin{aligned}
\operatorname{Inf}\left\{c(f), f^{*} \operatorname{Inf}\left\{c(g), g^{*} \tau_{z}\right\}\right\} & =\operatorname{Inf}\left\{c(f), f^{*} c(g), f^{*} \circ g^{*} \tau_{z}\right\} \\
& =\operatorname{Inf}\left\{\left\{\operatorname{Inf} c(f), f^{*} c(g)\right\},(g \circ f)^{*} \tau_{z}\right\} .
\end{aligned}
$$

Mais $\operatorname{Inf}\left\{c(f), f^{*} c(g)\right\}$ est la fonction de contrôle de la surjection composée $g$ of; on obtient ainsi l'expression voulue d'un tapis de $X$.

Les rapports entre les fonctions de contrôle $c(f)$ afférentes à diverses strates $X$ de $E$ sont ceux-là même qu'on exige d'un tapis: si $X$ et $Y$ sont deux strates de $E$ qui ont même image dans $E^{\prime}$, alors la restriction de $k_{Y X}$ aux variétés de niveau de $c(f)$ est de rang maximum; si $Z<Y<X$ est une chaîne de strates qui ont même image par $f$ les fonctions $k_{Y X}(c(f) \mid Y)$ et $c(f) \mid X$ ont des variétés de niveau qui se coupent transversalement dans $k_{Z X}^{-1}(z), z \in Z$. C'est dire qu'on peut utiliser les tapis Inf $\left.\} c(f), f^{*} \tau_{X^{\prime}}\right\}$ pour construire une présentation de $X$ adaptée au morphisme $f$ : l'image par $f$ d'une strate $[\omega]$ de la présentation est contenue dans la strate $\left[\omega^{\prime}\right]$ définie par la chaîne image; mais, en général, cette image est contenue dans $\left[\omega^{\prime}\right]$ comme sousvariété à bords (avec coins), et sa description exigera un raffinement de la stratification donnée sur le but $E^{\prime}$. Ainsi, dans une surjection stratifiée $f: E \rightarrow E^{\prime}$, il est en général impossible de munir $E$ et $E^{\prime}$ de présentations, telles que l'image par $f$ d'un élément de la présentation de $E$ soit un élément de la présentation de $E^{\prime}$. C'est le cas néanmoins lorsque $f$ appartient à une classe de morphismes particulièrement simples, les morphismes "sans éclatement," que nous définirons tout-àl'heure.

1. F. Espace tangent d̀ un ensemble stratifié. Soit $E$ un ensemble stratifié; à toute strate $X$ attachons son fibré des vecteurs tangents $T(X)$; soit $Y, X$ un couple de strates telles que $Y<X, L_{Y}(X)$ le lambeau d'incidence de $X$ relativement à $Y, k_{Y X}: L_{Y}(X) \rightarrow Y$ l'application d'attachement. On appellera espace tangent à $E$, noté $T(E)$, l'ensemble disjoint des $T(X)$ sur lesquelles on fait les identifications locales définies par les dérivées des applications d'attachement $T(X) \supset T\left(L_{Y}(X)\right) \stackrel{{ }^{1} k_{Y X}}{\longrightarrow} T(Y)$.

L'ensemble ainsi obtenu n'est évidemment pas compact; néan- 
moins les applications de recollement satisfont à la condition (Att); de plus, la surjection canonique $T(E) \rightarrow E$ peut être considérée comme un morphisme stratifié, les fonctions de contrôle étant données sur chaque strate $X$ par le module du vecteur tangent.

Définition 1.F.1. Sous-fibré du fibré des vecteurs tangents. On se donne, pour chaque strate $X$ de $E$, un sous-fibré vectoriel $A(X)$ de $T(X)$; on postule de plus, que, sur tout lambeau $L_{Y}(X)$, l'application $j^{1} k_{Y X}$ envoie $A(X)$ sur $A(Y)$.

La réunion des $A(X)$ définit-par passage au quotient défini par les $k_{Y X}$-un sous-fibré $A$ de $T(X)$.

Définition 1.F.2. Section d'un fibré tangent. Champ de vecteurs. Soit $A$ un sous-fibré du fibré des vecteurs tangents à $E$; une section de ce fibré est donnée pour toute strate $X$, par une section du fibré restreint à $X: s: X \rightarrow A(X)$; ces sections commutent aux applications de recollement $k_{Y X}$.

DÉfinition 1.F.3. Section contrôlée. Une section $s$ de $A$ est dite contrôlée, si, dans toute strate $X$, elle est tangente aux variétés de niveau d'un tapis $\tau_{X}$.

THÉORÈME 1.F.1. L'intégration d'un champ de vecteur $\xi$ contrôlé est possible, et permet de définir un groupe d un parametre d'homéomorphismes $h_{t}$ de $E$ sur lui-même.

DÉmonstration. L'intégration du champ $\xi$ est possible dans chaque strate $X$, car $\xi$ laisse invariantes les variétés compactes $\tau_{X}=\epsilon$; on pourra donc définir, dans chaque strate $X$, un groupe $h_{t}^{X}$ de difféomorphismes de $X$. Reste à montrer que, la réunion des $h_{t}^{X}$ passe au quotient dans $E$; en effet, un système fondamental de voisinages d'un point $x$ de $X$ dans l'étoile de $X$ peut être défini seulement en usant des tapis et des applications $k$ normalisées; comme le champ $\xi$ conserve et les tapis et les applications $k_{Y X}$, il transforme tout système fondamental de voisinages de $x \in X$ en un système fondamental de voisinages de $h_{t}(x) \in X$. (Rappelons qu'un tel système fondamental de voisinages est défini dans la section $k_{x}^{-1}(x)$ du tube $L X$ par des inégalités de la forme $k_{X_{i} X_{i}}^{*} \tau_{X_{i}}<\epsilon$, ainsi qu'on l'a vu dans la définition d'une présentation.)

ThÉoR ̇̀ME 1.F.2. Toute section contrôlée donnée sur un fermé de $E$ s'étend en une section contrôlêe sur $E$.

Ceci se démontre par induction sur la dimension des strates. Supposons la section donnée sur $X \cap F$ déjà définie sur le bord $\partial X$. On l'étend alors sur les lambeaux $L_{Y}(X)$ d'une présentation de $E$ : cette extension se fait par induction croissante sur la cohauteur d'une 
strate $Y \mathrm{du}$ bord; on a, à chaque pas, à étendre une section donnée sur le bord d'une variété à coins à l'espace ambiant, extension toujours possible.

Définition 1.F.4. Morphismes sans éclatement.

DÉfinition. Un morphisme stratifié surjectif $p: E \rightarrow E^{\prime}$ est dit sans éclatement, s'il satisfait à la condition suivante: étant donné un couple $X, Y$ de strates, $Y<X$, et $X^{\prime}, Y^{\prime}$ leurs images dans $E^{\prime}$, si l'on forme le diagramme commutatif:

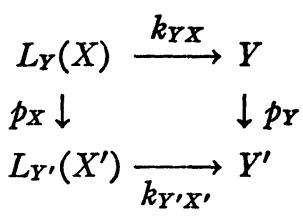

on en déduit, pour tout point $x \in L_{Y}(X), y=k_{Y X}(X)$, un homomorphisme $k: \operatorname{ker} p|T(x) \rightarrow \operatorname{ker} p| T(y)$ qui est alors une surjection.

Lorsqu'un morphisme $p$ est sans éclatement, le corang de $p$ sur une strate $X\left(=\operatorname{dim} X-\operatorname{dim} X^{\prime}\right)$ est supérieur au corang sur toute strate $Y$ du bord de $X$.

Une définition équivalente, mais plus intrinsèque, est la suivante: un morphisme surjectif $p: E \rightarrow E^{\prime}$ induit un homomorphisme $p$ du fibré des vecteurs tangents $T(E)$ dans le fibré $T\left(E^{\prime}\right)$. Si le noyau $\operatorname{Ker} p$ de cette application définit un sous-fibré de $T(E): 0 \rightarrow A \rightarrow T(E)$ $\rightarrow T\left(E^{\prime}\right) \rightarrow 0$ alors $p$ est sans éclatement.

Les propriétés suivantes sont de vérification immédiate:

(1.F.3) Le composé de deux morphismes sans éclatement est un morphisme sans éclatement.

(1.F.4) Si l'espace-but $E^{\prime}$ ne comporte qu'une strate, ou encore si $E^{\prime}$ ne comporte que deux strates $X^{\prime}, Y^{\prime}$ avec $\operatorname{dim} X^{\prime}$-dim $Y^{\prime}=1$, alors tout morphisme $p$ pour lequel l'application $k_{Y X}$ : Ker $p \mid T_{X}(x)$ $\rightarrow \operatorname{Ker} p \mid T_{Y}(y)$ de rang constant sur toute strate $X$, est un morphisme sans éclatement.

REMARQUE. Nous n'imposons pas la condition: $k_{Y X}: \operatorname{Ker} p \mid T(X)$ $\rightarrow \operatorname{Ker} p \mid T(Y)$ de rang constant sur toute strate $X$, pour un morphisme stratifié général. Cette condition peut sans doute être satisfaite, après un raffinement éventuel de la stratification, pour tout morphisme analytique, même avec éclatement. Les morphismes stratifiés pour lesquels cette condition ne pourrait être satisfaite au prix d'un raffinement des stratifications de la source et du but présentent un caractère hautement pathologique.

1. G. Les theorèmes d'isotopie. Soit $p: E \rightarrow E^{\prime}$ un morphisme stratifié surjectif, $U$ une strate de dimension $k$ du but $E^{\prime}$. Considérons 
une contre-image $p^{-1}(u)$ d'un point $u \in U$. Elle peut être munie canoniquement d'une structure stratifiée ainsi définie: Toute strate $X_{u}$ de $p^{-1}(u)$ est une intersection de la forme $X \cap p^{-1}(u)$; les lambeaux $L_{Y}\left(X_{u}\right)$ sont les restrictions à $p^{-1}(u)$ des lambeaux $L_{Y}(X)$, ainsi que les applications de recollement $k_{Y_{u} X_{u}}$ (rappelons que $k_{Y X}=p$ ). De même, le tapis de $X_{u}$ est la restriction à $p^{-1}(u)$ du tapis $\tau_{X}$. De manière plus générale, si l'on considère une présentation de $E$, son intersection avec $p^{-1}(u)$ donne une présentation de la contre-image. On va maintenant démontrer le

THÉORk̀ME 1.G.1. Dans tout morphisme stratifiê surjectif $E \stackrel{p}{\rightarrow} E^{\prime}$, la restriction de $p$ à la contre-image $p^{-1}(U)$ d'une strate $U$ de $E$ est une fibration.

Supposons d'abord que $U$ soit de dimension un (le théorème est trivial si $U$ est dimension zéro). Soit $V^{\prime}$ le champ de vecteurs unitaire sur $U$; sur tout segment $\left[u_{1} u_{2}\right] \subset U$, il est possible de relever le champ $V^{\prime}$ en un champ $V$, section contrôlée du fibré tangent $T(E)$, telle que $p V=V^{\prime}$. On observe que $\operatorname{ker} p \mid T(x) \rightarrow T(u), u=p(x)$, définit un sousfibré du fibré tangent à $p^{-1}(U)$. On construit alors une section contrôlée de ce sous-fibré (plus exactement, de l'ensemble des vecteurs $\phi$ tels que $p(\phi)=V^{\prime}$, champ unitaire) par induction sur la dimension des strates; sur les strates de dimension un de $p^{-1}(U)$, le champ $V$ est défini par sa projection $p$. Supposons le champ $V$ défini sur le bord d'une strate $X_{U}$; on construit alors la section $V$ par induction sur la cohauteur des strates $Y_{U}$ du bord; pour les strates de cohauteur un, les conditions $p(V)=V^{\prime}, k_{Y X}(V)=V, d \tau_{X}(V)=0$, définissent un sousfibré à fibre contractile de dimension positive sur le lambeau $L_{Y}(X)$. En effet, la première condition est conséquence de la seconde, et par suite la dimension de la fibre est $\operatorname{dim} X-\operatorname{dim} Y-1$, les autres conditions étant linéairement indépendantes en raison des hypothèses de surjection. Pour définir $V$ sur le lambeau associé à une strate de $Y$ de cohauteur $s$, on appliquera, comme toujours les propriétés standard des extensions différentiables de données sur les variétés à coins. L'intégration du champ $V$ dans $E$ fournit alors un champ d'homéomorphismes $h_{u}$ quit trivialise l'application $p \mid p^{-1}\left(u_{1} u_{2}\right) \rightarrow\left(u_{1} u_{2}\right)$, ce qui démontre la propriété.

Lorsque la strate $U$ est de dimension $k$, on fait choix sur le voisinage $W$ d'un point $u$, d'une famille radiale à $k-1$ paramètres de champs de vecteurs d'origine $u$ dans $U$, et on relève cette famille en une famille de champs de vecteurs $V$ dans la contre-image (ceci est possible, en raison des propriétés de continuité du prolongement des fonctions différentiables données sur une variété à coins (théorème 
de Seeley)). On intègre alors chaque champ relevé, et la famille de trivialisations ainsi obtenue définit une trivialisation globale de $p^{-1}(W)$ sur $W$. Ainsi s'achève la démonstration du Théorème 1.G.1.

Remarque. Dans un espace stratifié $E$, l'application d'un tube $L_{X}$ sur une strate $X$ par la rétraction $k$ est une application stratifiée dont les strates sont les lambeaux $L_{X}(Z), Z \in \mathrm{St} X$, les applications de recollement de $L_{X}(Z)$ sur $L_{X}(Y)$ lorsque $X<Y<Z$ étant données par les $k_{Y Z}$. De même, chaque lambeau a un tapis défini par les paramètres de la représentation sectorielle. On peut par suite appliquer le Théorème 1.G.1 à cette situation, ce qui montre que dans tout ensemble stratifié $E$ toute strate $X$ possède un voisinage fibré sur elle-même, la projection étant une rétraction par déformation $L_{X} \rightarrow X$.

Le second thêoreme d'isotopie. Theorème 1.G.2. Soit une suite de morphismes stratifiés $E \rightarrow E^{\prime} \rightarrow I$, où $p$ est sans éclatement, $I$ un segment muni de sa stratification triviale. Soient $a, b$ deux points intérieurs à $I$. Désignons par $E_{a}, E_{b}, E_{a}^{\prime}, E_{b}^{\prime}$ les sections de $E, E^{\prime}$ correspondant aux valeurs $t=a, t=b$ du paramètre $t$ défini sur $I$. Les applications sections $p_{a}: E_{a} \rightarrow E_{a}^{\prime}, p_{b}=E_{b} \rightarrow E_{b}^{\prime}$, sont des morphismes stratifiés sans éclatement pour les structures stratifiées définies sur les ensembles sections. On se propose de démontrer:

THÉOR ÈME 1.G.2. Les applications $p_{a}$, p ont même type topologique: il existe des homéomorphismes $h, h^{\prime}$ tels que le diagramme

$$
\begin{gathered}
E_{a} \stackrel{p_{a}}{\longrightarrow} E_{a}^{\prime} \\
h \downarrow \\
E_{b} \stackrel{p_{b}}{\longrightarrow} E_{b}^{\prime}
\end{gathered}
$$

commute.

De plus, $h$ et $h^{\prime}$ sont des isomorphismes stratifiés: l'homéomorphisme $h$ par exemple est induit d'une application stratifiée $E \rightarrow I$.

Lé démonstration repose sur le lemme suivant:

LEMME 1.G.3. Si $E \stackrel{p}{\rightarrow} E^{\prime}$ est un morphisme stratifié surjectif sans éclatement, on peut munir $E$ et $E^{\prime}$ de présentations telles que la présentation de $E^{\prime}$ soit un quotient de la présentation de E: l'image par $p$ de la variêté de symbole $[\omega]$ est la variêté de symbole $\left[\omega^{\prime}\right]$, où la chaîne $\left[\omega^{\prime}\right]$ $s^{\prime}$ obtient en remplaçant les strates $X$ de $E$ par leurs images $X^{\prime}$ dans $E^{\prime}$, et en identifiant éventuellement deux strates $X^{\prime}$ successives identiques.

On se donne "a priori" la présentation de $E^{\prime}$; on distingue dans $E$ des sous-objets stratifiés $E^{0} \subset E^{1} \subset \cdots E^{k}, E^{k}$ désignant l'ensemble- 
fermé -des strates où le corang de $p$ est inférieur à $k$. Une présentation de $E^{\prime}$ se relève de manière unique dans $E^{0}$; on définira la présentation de $E$ par induction croissante successive sur: (1) Le corang de $p$, (2) la dimension de $X$ pour un corang donné. Pour une strate $X$ donnée, il s'agit de définir les variétés de la présentation $[\omega X]$, cette présentation étant déjà définie sur les strates du bord de $X$. On procède ainsi: pour toute strate $Z$ du bord de $X$, on définit d'abord un "lambeau normalisé" $\Lambda_{Z}(X)=\mathrm{U}_{\omega}[Z \omega X]$, où $\omega$ parcourt l'ensemble des chaines $\omega$ telles que $Z \omega X$ soit une chaîne. Une fois qu'on a défini tous les $\Lambda_{Z}(X)$, on obtient les éléments de la présentation par intersection: $[\omega X]$ est l'intersection $\bigcap_{i} \Lambda\left(Z_{i} X\right)$, où $Z_{i}$ parcourt la chaîne $\omega$.

La démonstration précède par induction sur la cohauteur de la strate $Z$ relative à $X$. Supposons qu'on ait pu "normaliser" l'attachement de la strate $X$ sur les strates de cohauteur $<s$. Ceci veut dire, rappelons-le, qu'on peut définir $X$ comme l'intérieur d'une variété à bord à coins $P$, difféomorphe à $\operatorname{Inf}\left(\tau_{x}, g_{X}\right)>\epsilon$, de bord $V$, et qu'on a pu définir, pour toute strate $Z^{\prime} \in \mathrm{St} Z$ et incidente à $X$, une application d'attachement $h: k^{-1}\left(Z^{\prime}\right) \rightarrow{ }^{n^{v}} Z^{\prime}$, différentiable par morceaux, voisine de $k_{Z^{\prime} X}$ sauf au bord de $Z^{\prime}$, telle que la contre-image de toute strate $Z^{\prime}$ par $h$ soit une variété à coins dans $V$. L'application $h$ peut être étendue globalement à l'extérieur d'un voisinage de $Z^{\prime}$. Comme on l'a vu, en 1.D., cette normalisation permet, par la formule $h^{-1}[\omega]$ $=[\hat{\omega} X]$ d'étendre la présentation au-dessus des strates de cohauteur $<s$ car on a encore $k_{Y X}\left[\omega^{\prime} \hat{Y} X\right]=\left[\omega^{\prime} Y\right]$, pourvu que la longueur de la chaîne $\omega^{\prime}$ n'excède pas $(s-1)$.

Supposons d'abord que $p(Z)=p(X)$. Alors toutes les strates $Y$ telles que $Z<Y<X$ ont $p(X)$ pour projection. Toutes les applications $k_{Z Y}, k_{Y X}$ sont "verticales." En normalisant l'ensemble des $\left(k_{Y X}\right)$ audessus du tube $L_{Z}$, on les remplace par une application $h$ localement verticale. La procédure du théorème I.D.1 s'applique telle quelle et permet d'étendre la présentation au-dessus de la strate $Z$, si elle est donnée sur les strates de l'étoile $L_{Z}$. Le bord du lambeau $\Lambda_{Z}(X)$ est alors défini par une variété de niveau $g_{X}=\epsilon$ de la fonction de contrôle $g$ de la submersion $X \rightarrow X^{\prime}=p(X)$.

Passons maintenant au cas général $Z^{\prime}=p(Z)<X^{\prime}=p(X)$. On forme, comme précédemment le tube $L_{Z}$ de la strate $Z$ réunion des lambeaux $\Lambda_{Z}(U)$ où $Z<U<X$. L'image par $p$ de $L_{Z}$ est le tube $L_{Z^{\prime}}=U \Lambda_{Z^{\prime}}(U)$ où $Z^{\prime}<U^{\prime}<X^{\prime}$. Soit $t$ le rayon du voisinage tubulaire $L_{Z^{\prime}}$ de $Z^{\prime}$ dans $L_{Z^{\prime}}, \partial L_{Z^{\prime}}$ le bord $t=1$. Par l'hypothèse d'induction, on peut remplacer le système des $k_{U X}$ par une application d'attachement $h$ définie sur une portion $A$ de $V$, à valeur dans $L_{z}$, qui se projette sur 
une application $h^{\prime}$ d'attachement de $X^{\prime}$ sur $Z^{\prime}$. Or cette portion $A$ est évidemment située dans $X \cap p^{-1}\left(h^{\prime-1}\left(\partial L_{Z^{\prime}}\right)\right)$, car l'application d'attachement $h$ est "verticale" par construction sur la portion de la forme $\left\{g_{x}^{\prime}=\epsilon\right\}$ de $V$. On devra donc considérer dans $X \cap p^{-1}\left(h^{\prime-1}\left(\partial L_{Z^{\prime}}\right)\right)$ une contre-image de la forme $h^{-1}\left(\partial L_{Z}\right)$ pour un rayon $\epsilon=s(t)$ du tube $L_{Z^{\prime}}$. Ceci définit dans $p^{-1}\left(h^{\prime-1}\left(\partial L_{Z^{\prime}}\right)\right) \cap X$ une hypersurface à coins $H_{!}^{\prime}$ d'intérieur $H_{\mathbf{e}}$ dont toutes les faces sont appliquées par $k_{Z X}$ dans $Z$ avec rang maximum. (Ici intervient l'hypothèse de non éclatement.) On pourra donc par normalisation étendre l'application $h$ au-dessus de la strate $Z$ avec $h=k_{Z X}$ de telle manière que la contre-image $h^{-1}(Z)$ soit la variété à coins $H_{\epsilon}, h \mid H_{\mathrm{e}} \rightarrow Z$ étant une fibration; la projection $p\left(H_{\epsilon}\right)$ est $h^{\prime-1}\left(\partial L_{Z^{\prime}}\right)$ soit la strate $\left[\hat{Z}^{\prime} X^{\prime}\right]$ de la présentation du but: la présentation en secteurs du but $E^{\prime}$ permet de définir dans $L_{Z^{\prime}}\left(X^{\prime}\right)$ un champ de vecteur $\xi$ définissant la rétraction $k_{Z^{\prime} X^{\prime}}^{\prime}$ (et divers autres champs $\xi_{i}$ pour les rétractions $k_{Y^{\prime} X^{\prime}}$ ). On définira la variété $[Z X]$ par la réunion des variétés $H_{\epsilon}$ pour $\epsilon \rightarrow 0$. Les divers champs $(\xi)$ de la représentation sectorielle du but se relèvent en champs des rétractions correspondantes car toutes les constructions précédentes peuvent être faites dans une contre-image de la forme $p^{-1}(\xi) \cap X$. Ceci permet d'étendre à $X$ la représentation sectorielle de l'espace source $E$ définie au Lemme 1.G.3. Les variétés $[Z U X]$ pour $Z<U<X$ sont définies comme en I.D., ainsi que les injections $[Z O X] \rightarrow[Z X]$. Les relations d'injection des variétés à coins de type $[\hat{Z} O X] \rightarrow[\hat{Z} X]$ passent alors au quotient dans $E^{\prime}$. On se contentera ici de ces indications, dont l'explicitation exigerait un déploiement symbolique considérable. Voir Figure 5 pour un cas simple de cette construction. Le Lemme 1.G.3. étant admis, on démontre ainsi le Théorème 1.G.2: Soit $\partial / \partial_{t}$ le champ unitaire sur $I$; dans la contre-image $a \leqq t \leqq b$ dans $E^{\prime}$, il existe un champ $V^{\prime}$ dont l'image par $t$ est $\partial / \partial_{t}$ (Théorème 1.G.1); on montre qu'il existe un champ de vecteurs $V$ sur $E$, tel que $p(V)=V^{\prime}$, qui forme une section contrôlée de $T(E)$, ou, plus exactement, du sous-fibré $\operatorname{Ker} p$ de $T(E)$. L'existence d'une telle section se montre par récurrence sur le corang de $p$ d'abord, puis sur la dimension $X$ de la strate. Dans les lambeaux "horizontaux," $L_{Z}(X)$, le champ $V$ est assujetti à la seule condition de commuter avec $k_{Z X}$; dans les lambeaux verticaux, $L_{Y}(X)$, on lui impose d'admettre les fonctions de contrôle $g$ comme intégrales premières. Ceci définit sur la réunion de ces lambeaux des systèmes de fibrés vectoriels emboîtés de dimension positive, croissant avec la cohauteur de la strate $Y$ (ou $Z$ ); on étendra donc le champ $V$ par le théorème de prolongement relatif aux variétés à coins. On obtient finalement un champ $V$ qui laisse invariant, en 


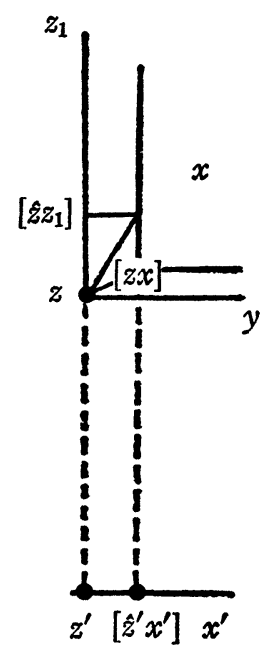

FIGURE 5

toute strate $X$, la fonction de contrôle $g$, et l'image $p^{*} \tau_{X^{\prime}}$ du tapis de la strate image. Il définit par suite une section contrôlée de $T(E)$ qu'on pourra intégrer, ce qui permettra de définir l'homéomorphisme $h$, comme l'intégration de $V^{\prime}$ définit $h^{\prime}$.

DÉFinition 1.G.1. Ensembles stratifiés isomorphes. Deux ensembles stratifiés $E_{1}, E_{2}$ sont dits isomorphes, s'il existe un ensemble stratifié $A$ et un morphisme stratifié $A \rightarrow I$, tel que $E_{1}$ soit la section $t=a, E_{2}$ la section $t=b$ de ce morphisme. Ceci défini entre ensembles stratifiés une relation d'équivalence; en effet, en accollant deux tels produits le long de leur face $t=b$ commune, on peut lisser les applications $t$ et $k$ le long des strates de cette face par l'artifice classique qui consiste à "épaissir" cette face en $t$ (plus exactement effectuer sur $t$ un difféomorphisme localement plat...). On pourrait évidemment ne considérer que les classes modulo cette relation d'équivalence, ce qui permettrait d'éliminer de la définition d'un ensemble stratifié les tapis, les lambeaux et les $k_{Y X}$ pour n'en conserver qu'une classe d'isotopie globale.

\section{Théorie Euclidienne}

\section{A. Preliminaires. Angle d'un p-plan et d'un $q$-plan dans $\boldsymbol{R}^{n}$.}

Définition 2.A.1. Soient $A^{p}, B^{q}$ un $p$-plan, un $q$-plan issus de l'origine 0 de $R^{n}$. Supposons $p \leqq q$, et désignons par $G_{q}$ la grassmannienne des $q$-plans issus de 0 ; l'ensemble des $q$-plans contenant le $p$-plan $A^{p}$ forme une sous-variété (cycle de Schubert) $Z\left(A^{p}\right)$ dans $G_{q}$. On appellera angle intérieur du couple $A^{p}, B^{q}$ la distance, notée 
$d\left(A^{p}, B^{q}\right)$, du point $B^{q}$ à $Z\left(A^{p}\right)$ dans $G_{q}$. Dire que $d\left(A^{p}, B^{q}\right)=0$, c'est dire que $A^{p}$ est contenu dans $B^{q}$.

DÉfinition 2.A.2. Supposons maintenant $p+q \geqq n$; soit, dans la grassmannienne $G$, l'ensemble $Z^{\prime}\left(A^{p}\right)$ constitué des $p$-plans non transverses à $B^{q}$; c'est ici encore un cycle de Schubert de $G_{q}$. On appellera angle extérieur du couple $A^{p}, B^{q}$ la distance notée $D(A, B)$ du point $B^{q}$ à l'ensemble $Z^{\prime}\left(A^{p}\right)$ dans $G_{q}$. Dire que $D\left(A^{p}, B^{q}\right)=0$, c'est dire que $A^{p}$ et $B^{q}$ ne sont pas transverses (sont contenus dans un sous-espace $\boldsymbol{R}^{p}$ de $R^{n}$ ).

Citons enfin le lemme suivant, qui ne fait qu'exprimer la continuité de l'opération: Intersection transversale:

LEMME 2.A.1. Si $A_{p}, B_{q}, p+q \geqq n$, sont deux plans variables fonctions d'un paramètre $t$ tendant vers zéro, et si dans un voisinage de $t=0$, l'angle extérieur $D\left(A_{p}, B_{q}\right)$ est minoré par une constante positive $C>0$, alors

$$
\lim _{t=0} A_{p} \cap B_{q}=\lim _{t=0} A_{p} \cap \lim _{t=0} B_{q}
$$

quand ces limites existent. Dans le cas général, où l'on a, non une limite unique, mais un ensemble limite, il y a alors inclusion:

$$
\lim _{t=0} A_{p} \cap B_{q} \subset\left\{\lim _{t=0} A_{p}\right\} \cap\left\{\lim _{t=0} B_{q}\right\} .
$$

Les propriêtés $A, B$ de Whitney. Soit $X$ une variété plongée de dimension $q$ dans $R^{n}, Y$ une autre variété de dimension inférieure $p<q$. On note $T_{x}(X)$ l'espace des vecteurs tangents à $X$ au point $x$. Soit $y$ un point de $Y$ adhérent à la variété $X$. On dit que $X$ présente en $y$ la propriété (A) par rapport à $Y$ si:

$$
\lim _{x y=0} d\left(T_{y}(Y), T_{x}(X)\right)=0 .
$$

De même, $X$ présente en $y$ la propriété (B), si pour tout couple de points $x$ dans $X, y^{\prime}$ dans $Y$ tendant vers $y$

$$
\lim _{x y=0 ; y, y=0} d\left(y^{\prime} y, T_{x}(X)\right)=0 .
$$

Dans la pratique, on substituera à (B) une propriété $\left(\mathrm{B}^{\prime}\right)$ ainsi définie; soit $k$ une retraction locale d'un voisinage tubulaire $T_{\boldsymbol{Y}}$ de $Y$ sur $Y$; on pose alors $y^{\prime}=k(x)$, et $\left(\mathrm{B}^{\prime}\right)$ s'écrit:

$$
\lim _{x y=0} d\left(y^{\prime} y, T_{x}(X)\right)=0 .
$$

Si, sur un voisinage de $y$ dans $Y$, la propriété (A) est satisfaite, alors 
(B) et $\left(\mathrm{B}^{\prime}\right)$ sont équivalentes, et le choix de la rétraction $k$ est indifférent.

Voisinage tubulaire d'une variêté plongée. Fonction tubulaire. Soit $X$ une variété $C^{\infty}$ proprement plongée dans $R^{n}$ de codimension $k$; à tout point $x \in X$, on peut associer une carte locale $U\left(x_{i}, u_{j}\right)$, $i=1, \cdots, n-k, j=1, \cdots, k$ dans laquelle $X \cap U$ est défini par les équations locales $u_{j}=0, j=, 1 \cdots, k$.

DÉFINITION 2.A.3. On appellera fonction tubulaire pour $X$ une fonction $g C^{\infty}$, définie sur un voisinage ouvert de $X$, telle qu'en tout point $x$ de $X$, il existe une carte adaptée dans laquelle $g$ s'écrive $g=\sum_{j=1}^{k} u_{j}^{2}$.

On impose de plus à $g$ les conditions suivantes; soit $k(x)$ un champ de $k$-plans transverses à $X$; ils définissent une rétraction, notée $k$ d'un voisinage tubulaire $T(X)$ sur $X$. Si l'on considère l'ensemble $S$ défini par $g \leqq \epsilon$, cet ensemble est fibré par $k$ en $k$-boules fermées sur $X$; de plus, l'adhérence $\bar{S}$ est telle que $\bar{S}=\bar{X}-X$ dans $R^{n}$. On vérifie immédiatement que l'ensemble des fonctions tubulaires pour $X$ est non vide (par recollement par une partition de l'unité) et convexe: si $g$ et $h$ sont tubulaires, la fonction $a f+b g, a, b>0$, est aussi tubulaire; de même la fonction $f(y) \cdot g$, si $f$ est plus grande que un sur $X$.

\section{B. Objets de Whitney.}

Définition 2.B.1. On appellera objet de Whitney ( $W$-objet) un sous-espace $E$ de $R^{n}$ qui est une réunion de variétés proprement plongées $X_{i}$, les strates de $E$. On fait sur leur attachement l'hypothèse suivante: (W) Si $Y \cap \bar{X}$ est non vide, alors $\operatorname{dim} Y<\operatorname{dim} X$, et, en tout point de $Y \in \bar{X}$, les propriétés (A) et (B) sont vérifiées.

On ajoutera également la condition suivante:

(Nf) Tout voisinage tubulaire $T(X)$, assez petit, d'une strate $X$ ne rencontre qu'un nombre fini de strates $X_{j}$.

On se propose d'établir le

\section{THÉORèME 2.B.1. Tout $W$-objet compact est un ensemble stratifié.}

Il est clair que la condition (Nf) entraine qu'un $W$-objet compact ne comporte qu'un nombre fini de strates. Car, s'il existait dans $E$ un nombre infini de strates de dimension $k$, la frontière de leur réunion ne pourrait comporter que des strates de dimension strictement inférieure à $k$, elles-mêmes en nombre infini; et ainsi de suite jusqu'aux strates de dimension zéro, qui ne peuvent être en nombre infini, à cause de (Nf) et de la compacité de $E$. Il suffira par suite de démontrer le Théorème 2.B.1 par induction sur le nombre des strates, elles- 
mêmes ordonnées par dimension croissante. De manière plus précise, on démontrera par induction:

$\left(1^{\circ}\right)$ La propriété de frontière. Si $Y \cap \bar{X}$ est non vide, alors $Y$ connexe - est toute entière contenue dans $\bar{X}$, ce qu'on écrira $Y<X$ ( $Y$ incidente à $X$ ). Cette relation est transitive: $Y<X$ et $Z<Y$ impliquent $Z<X$.

$\left(2^{\circ}\right)$ A toute strate $X$, on associera une fonction tubulaire $g_{X}$ et un tube $T_{X}$, tels que $T_{X}$ et $T_{Y}$ ne se rencontrent que si $Y<X$ ou $X<Y$.

$\left(3^{\circ}\right)$ A toute chaine de strates $X_{1}<X_{2}<\cdots<X_{k}$ se trouve associée l'intersection transversale des bords des tubes

$$
g_{X_{1}}=\epsilon_{1}, \quad g_{X_{2}}=\epsilon_{2} \cdots g_{X_{k}}=\epsilon_{k} .
$$

$\left(4^{\circ}\right)$ A toute strate $X$ se trouve associé un champ de plans transverses $k_{x}$ qui définit par restriction des rétractions $k_{X}: T_{X} \rightarrow X$. L'intersection $T_{Y} \cap X$ définit le lambeau $L_{Y}(X)$ si $Y<X$. Le champ $k_{x}$ doit évidemment satisfaire à la condition (Trans) des recollements: Sur $L_{Y}(X)$, on a $k_{x} \subset k_{Y}^{-1}\left(k_{Y}(x)\right)$.

$\left(5^{\circ}\right)$ Les tapis $\tau_{X}$ de chaque strate $X$ sont définis par lissage des fonctions $g_{Y}$ des strates $Y$ du bord $\partial X$.

L'induction démarre de façon évidente sur les $W$-objets compacts de dimension zéro, constitués d'un nombre fini de points. Un objet constitué d'une seule strate est une variété plongée compacte. On va faire une démonstration explicite pour un $W$-objet $E$ constitué de deux strates $X, Y$. Soit $Y<X, Y$ compacte. On forme un voisinage tubulaire $L_{Y}$ de $Y$, défini par la fonction tubulaire $g_{Y}$ avec une rétraction transverse définie par le champ de $k$-plans $k_{Y}(y)$.

Etablissons d'abord la propriété de frontière: $Y \cap \bar{X}=Y$. Pour cela, considérons l'ensemble $Y \cap \bar{X}=Z ; Z$ est visiblement fermé dans $Y$, puisque $\bar{X}$ est compacte. Montrons que $Z$ est également ouvert; soit $z$ un point de $Z$ adhérent à $Y-Z$ supposé non vide; au voisinage de $z$, il existe des points $y$ adhérents à $X$ : à tout $y$ de ce type, on peut associer un $\epsilon$ tel que l'application locale $k_{Y}$, restreinte à $g_{Y}=\epsilon$, contienne $y$ dans son image; or, la même propriété ne peut être vraie du point $z_{1} \in Y$ qui n'appartient pas à $Z$, donc n'est pas adhérent à $X$. Il en résulte que tout chemin $c$ joignant $y$ à $z_{1}$ rencontre l'ensemble des valeurs critiques des applications $k_{Y} \mid g_{Y}=\epsilon$, et ceci si petit que soit $\epsilon$, et si proches que soient $y$ et $z_{1}$ de $z$. C'est dire qu'un tel point $z$ est adhérent à l'ensemble des points critiques des restrictions $k_{Y} \mid g_{Y}$ $=\epsilon, \epsilon \rightarrow 0$; or, une telle propriété est exclue pour un point où les propriétés (A) et (B) sont vraies pour l'in lusion $Y \subset \bar{X}$. En effet, désignons par $T g_{y}(x)$ le plan tangent au bord du tube $g_{y}=\epsilon$ dans $R^{n}$. Pour 
$x$ tendant vers $y$, on applique le Lemme 2.A.1 au couple de plans $T_{x}(X), T g_{y}(x)$. En raison de la propriété (B), l'angle extérieur $D\left(T_{x}(X), T g_{Y}(x)\right)$ est minoré par une constante fixe $>0$; mais $T_{y}(Y)$ est contenu dans $\lim T_{x}(X)$ (Propriété A); de même $T_{y}(Y)$ est contenu dans $\lim T g_{Y}(x)$ : propriété immédiate des fonctions tubulaires; par suite $T_{y}(Y)$ est contenu dans toute limite $T_{x}(X) \cap T g_{Y}(x)$, ce qui montre que $k_{Y}$ est de rang maximum sur les variétés de niveau de $g_{Y}=\epsilon$ dans $X$ pour $\epsilon$ assez petit.

La propriété de frontière $Y \cap \bar{X}=Y$ étant ainsi établie, on définit le lambeau $L_{Y}(X)$ par l'intersection $L_{Y} \cap X$, l'application d'attachement $k_{Y X}$ par la restriction de $k_{Y}$ à $L_{Y}(X)$.

Un tapis $\tau_{X}$ s'obtient en restreignant $g_{Y}$ à $X$. Pour pousser plus loin l'induction, il est nécessaire de définir un champ transverse $k$ à $X$; on impose à ce champ d'être contenu dans le plan $k_{Y}^{-1}\left(k_{Y}(x)\right)$ en tout point de $L_{Y}(X)$; comme l'ensemble des directions de plans ainsi défini en chaque point de $L_{Y}(X)$ forme un ouvert contractile d'une certaine grassmannienne ( $G_{p}^{q}$ où $p=\operatorname{codim} X, q=\operatorname{codim} Y$ ), il existe un tel champ de plans. On l'étend ensuite à tout $X$, ce qui est possible, toujours à cause du fait que l'ensemble des $p$-plans transverses à une variété linéaire de codimension $p$ est contractile. Et, bien entendu cette extension peut être faite différentiablement sur $X$. On définira ensuite la fonction tubulaire $g_{X}$ et le tube $L_{X}$ par les deux conditions: (i) $K_{X}$, la rétraction linéaire définie par $k_{X}$ sur les tubes $g_{X}=\epsilon$ est une surmersion. (ii) Les tubes $g_{X}=\epsilon$, et $y_{Y}=\epsilon^{\prime}$ se coupent transversalement dans un voisinage de $Y$. On a vu plus haut que les tubes $g_{Y}=\epsilon^{\prime}$ rencontrent transversalement la strate $X$; elles rencontrent donc transversalement tous les tubes $g_{X}=\epsilon_{x}$ pour un $\epsilon_{x}$ dépendant de $x$. Mais, en fait, on pourra obtenir un $\epsilon$ indépendant de $x$ en multipliant éventuellement la fonction $g_{X}$ par une fonction positive de la forme $a\left(g_{Y} k_{X}(x)\right)$. Ceci ne modifie pas en effet les propriétés de transversalité des intersections $g_{\bar{X}}^{-1}(\epsilon), g_{\bar{Y}}^{-1}\left(\epsilon^{\prime}\right)$, car $\operatorname{grad}\left(a g_{Y}\right) \cdot g_{X}=a \operatorname{grad} g_{X}$ $+a^{\prime} \operatorname{grad} g_{Y} \cdot g_{X}$, et ceci n'affecte pas le produit extérieur grad $g_{X}$ $\wedge \operatorname{grad} g_{Y}$. (Ce calcul suppose, ce qu'on peut réaliser par une métrique riemannienne convenable, que les plans de rétraction $k_{\bar{X}}^{-1}(x)$ sont localement dans les variétés de niveau $g_{Y}=$ cste.)

Nous pouvons maintenant aborder l'étape majeure de l'induction. On se donne un objet de Whitney $E=A \cup U$, où $U$ est une strate de dimension maximum; $A$ est la réunion des autres strates de dimension inférieure à la dimension $u$ de $U$; on admet, par induction, que $A$ possède une structure stratifiée définie par des voisinages tubulaires des strates et des rétractions $k_{Y}$ définies par des champs de plans transverses. 
On démontre d'abord que la propriété de frontière est vraie: si $Y$ est une strate qui rencontre $\bar{U}$, alors $Y \cap \bar{U}=Y$. Supposons d'abord que $Y$ soit de dimension maximum parmi toutes les strates qui rencontrent $\bar{U}$; alors, au voisinage d'un point $y$ de $Y$, la fonction $g_{Y}$ est localement propre sur $U$; s'il n'en était pas ainsi, il existerait des points de $\bar{U}-U$, non situés dans $Y$, et arbitrairement voisins de $y$; ces points appartiendraient donc à une strate $X$ de $A$ rencontrée par le tube $L_{Y}$ de $Y$, donc de dimension supérieure à $Y$, contrairement à l'hypothèse. On pourra donc faire pour l'injection $Y \subset \bar{U}$ le même raisonnement que celui fait plus haut dans le cas de l'ensemble à deux strates.

La propriété s'étend ensuite aux autres strates de dimension plus petite, par induction sur la "cohauteur" de la strate. Soit $Z$ une telle strate, de cohauteur $s$. On considère dans l'étoile de $Z$ l'ensemble $V$ des strates qui rencontrent $\bar{U}$. La rétraction $k_{z}$, restreinte aux variétés de niveau de $g_{Z}$ n'a pas de points critiques, sauf éventuellement dans un voisinage de $V$; au voisinage d'une strate $X$ de $V$, on a $k_{Z}=k_{Z X} \circ k_{X}$; dans le lambeau $L_{X}(U)=U \cap L_{X}$, on substitue à la fonction $g_{Z}$ la fonction $g_{X}$; comme ces diverses fonctions $g_{X}$ ont des différentielles linéairement indépendantes, on peut définir une fonction à coins du type local $\operatorname{Inf}\left(g_{z}, g_{X_{1}}, \cdots, g_{X}\right)$, où on a au plus $s$ fonctions $g_{X_{i}}$, qui sert à définir un tapis local pour $U$. Or, la rétraction locale $\boldsymbol{k}_{Z}$ est elle aussi de rang maximum sur les variétés à coins définis par ce tapis (là où on a un coin, $k_{z}$ est de rang maximum sur toutes les hypersurfaces qui définissent le coin, ainsi que sur leurs intersections). On le voit en usant des identités $\boldsymbol{k}_{Z}=\boldsymbol{k}_{Z X} \circ \boldsymbol{k}_{X}$. La démonstration faite pour le cas de deux strates s'étend au cas général, en remplaçant la fonction $g_{z}$ par ce tapis local à coins, qui est localement propre, et pour lequel on peut refaire le raisonnement sur l'ensemble critique de $k_{z}$.

Une fois la propriété de frontière établie, on définit les lambeaux et les $k_{X}$ par: $L_{Z}(U)=L_{Z} \cap U ; \boldsymbol{k}_{Z U}=\boldsymbol{k}_{Z} \mid L_{Z}(U)$. Un tapis pour $U$ est défini par une fonction à coins du type local $\operatorname{Inf}\left(g_{X_{1}}, g_{X_{2}} \cdots g_{X_{8}}\right)$, où les $X_{1} X_{2} X_{s}$ forment une chaîne de strates aboutissant à $U$, cette expression étant valable dans l'intersection $L_{X}(U) \cdots L_{X_{i}}(U)$. On pourra éventuellement lisser cette fonction à coins si l'on veut des tapis $C^{\infty}$. Il reste, pour achever l'induction, à montrer qu'on peut associer à $U$ un tube $L_{U}$, une fonction tubulaire $g_{U}$, et un champ de rétractions linéaires locales $k_{U}$.

Pour définir $g_{U}$, on part d'une fonction tubulaire arbitraire pour $U$, et on affine le tube défini par $g<\epsilon$, en multipliant $g$ par une fonction positive de la distance au bord de $U$. Ceci afin que les tubes $g_{U}=$ cste 
rencontrent transversalement les tubes $g_{X_{i}}$ et leurs intersections dans les "secteurs" $L_{X_{1}}(U) \cap L_{X_{2}}(U) \cdots$.

La fonction "distance au bord" peut être par exemple le tapis de $U$, après lissage. (Observer que le gradient d'une fonction lissée d'une fonction à coins est combinaison linéaire à coefficients positifs des gradients des fonctions définissant le coin.) La démonstration du cas de deux strates sur le produit extérieur grad $g_{X} \wedge \operatorname{grad} g_{Y}$ s'étend par suite à un produit extérieur multiple de la forme grad $g_{X_{1}} \wedge \operatorname{grad} g_{X_{2}}$ $\wedge \cdots \wedge$ grad $g_{U}$, lorsque $\left\{X_{1} \cdots X_{i} \cdots U\right\}$ est une chaîne de strates.

La construction du champ $k_{U}$ fait appel à une construction par prolongement de sections d'un fibré différentiable à fibre contractile. On construit $k_{U}$ d'abord sur les lambeaux $L_{X}(U)$, où $X$ est de cohauteur un: la condition $k_{U}(u) \subset \boldsymbol{k}_{\bar{X}}^{-1}\left(\boldsymbol{k}_{X U}(u)\right)$ définit sur $L_{X}(U)$ un fibré dont la fibre set un ouvert contractile de la grassmannienne; on en construit une section, qu'on étend ensuite aux lambeaux $L_{Z}(U)$ selon la cohauteur croissante de $Z$; à chaque pas, on a à étendre une section déjà donnée sur le bord d'une variété à coins à l'intérieur de cette variété, ce qui est possible par prolongement standard.

La construction ici présentée fournit pour $U$ un tapis à coin et un système de rétractions $k_{X_{i}}$. Il reste si l'on veut obtenir $E=A \cup U$ comme ensemble stratifié, à définir une application d'attachement globale du bord de la variété $g=\epsilon$ dans $A$. On peut le faire, d'abord en lissant le tapis $\tau$, ensuite en recollant les diverses applications $\boldsymbol{k}_{X_{i}}$ en une application $h$ unique. En utilisant la notation d'une présentation, on refera l'opération inverse de la "normalisation" décrite en \$1.D. Si $Z<X<U$, par exemple, on peut modifier $k_{X U}$ en $k_{X U}^{\prime}$ dans un voisinage tubulaire de la variété de symbole $[\hat{Z} \hat{X} U]$ de manière à ce que le système $k_{X V}^{\prime}, k_{Z U}$ soit continu sur [ $\left.\hat{Z} \hat{X} U\right]$; après une telle suite d'opérations, on aura une application d'attachement $h: g^{-1}(\epsilon)$ $\rightarrow A$, globalement continue, et telle que sa composée avec une $\boldsymbol{k}_{X_{i}}$ déjà donnée sur $A$ soit une submersion sur $X_{i}$. On retrouvera ainsi notre définition initiale des ensembles stratifiés (\$1.C). De même, la structure de présentation s'obtient immédiatement, par la règle suivante: la variété de symbole $\left[X_{1} X_{2} \cdots U\right]$ est l'intersection de $U$ et des tubes $L_{X i}$; à un symbole $\hat{X}_{j}$ correspond le bord du tube $\partial L_{j}$. La structure en secteurs est réalisée par des "géodésiques" normales ou des gradients, par rapport à des métriques riemannienne locales convenablement adaptées; nous n'insisterons pas davantage sur ce point qu'un lecteur patient saura reconstituer.

En dépit du résultat obtenu par le Théorème 2.B.1, la structure d'un $W$-objet peut être relativement pathologique; ainsi, dans le plan de coordonnées polaires $(r, \theta)$, la spirale d'équation polaire 
$r=\exp -\theta^{2} / 2$. L'angle $V$ de la spirale avec le rayon vecteur est donné par tg $V=r d \theta / d r=-1 / \theta$. Cet angle tend vers zéro quand $\theta$ tend vers l'infini, donc quand $r$ tend vers zéro. L'ensemble constitué d'un $\operatorname{arc} L$ de la spirale limité à $0<0<\infty$, et compactifié par l'origine 0 est un $W$-objet (avec la stratification définie par l'arc $L$ comme 1 -strate, et l'origine 0 comme zéro-strate). En effet, les conditions (A) et (B) sont satisfaites en 0 (A trivialement, $B$ à cause de $\lim _{-0} V=0$ ). Il est clair qu'une telle disposition spiralée ne peut être présentée par un ensemble analytique en 0 . Pour un tel ensemble, en effet l'angle $V$ admet une majoration hölderienne $V<K_{0} r^{1 / \alpha}, 0<\alpha$ qui assure la convergence de l'intégrale $\theta=\int_{r_{0}}^{0} d r \operatorname{tg} V / r$.

C. Projection d'un $W$-objet. L'image par une projection linéaire $p: \boldsymbol{R}^{n+k} \rightarrow \boldsymbol{R}^{n}$ d'un $W$-objet compact de $\boldsymbol{R}^{n+k}$ n'est pas, en général, un $W$-objet: la présence d'étoiles spiralées introduit en effet une infinité de strates de valeurs critiques. On va se placer en un cas très particulier, qui se présente toutefois en de nombreuses applications.

Soit $E$ un $W$-objet compact situé dans $R^{n+1}, t$ l'application linéaire $t=R^{n+1} \rightarrow R$ définie par la dernière coordonnée. Supposons que la fonction $t$ soit de rang un $(d t \neq 0)$ sur toutes les strates de $E$ de dimension supérieure à un. L'image par $t$ de $E$ est un segment $J$ de $R$, comportant comme strates de dimension zéro les points images des strates de dimension zéro de $E$. Munissons $J$ de la stratification évidente définie par ces points comme zéro-strates. On a alors le:

THÉOR̀̀me 2.C.1. Il existe-sous les conditions énoncées ci-dessousune structure stratifiée sur $E$ telle que la projection $t: E \rightarrow J$ soit un morphisme stratifié sans éclatement.

On commence par raffiner la stratification donnée sur $E$ en introduisant, pour toute strate $X$ initiale, les contre-images $t^{-1}\left(q_{i}\right) \cap X$ des points $q_{i}$ de la stratification intérieurs à $J$. L'introduction de ces nouvelles strates ne change pas le caractère de $W$-objet de $E$, car les propriétés (A) et (B) se conservent par section transversale. Il n'en va pas de même pour l'injection d'un sommet $a \in E$ dans une nouvelle strate $X \cap t^{-1}(t(a))$; là, la propriété (B) n'est pas automatiquement vérifiée, et doit faire l'objet d'une hypothèse spéciale (section transversale de l'étoile de $a$ ): ce sera le cas, notamment, si l'angle externe $D$ du plan $t=$ cste avec le plan tangent $T_{x}(X)$ est minoré par une constante positive au voisinage de $a$.

Ceci étant admis, on définit la structure stratifiée de $E$ par la procédure utilisée pour un $W$-objet, avec la seule restriction supplémentaire que si $X$ et $Y$ sont deux strates de même projection sur $J$, alors le champ de plans $k_{Y X}$ est "vertical," contenu dans Ker $t$. En 
fait, on pourra prendre pour tous les $k_{X}$ des champs "verticaux" (contenus dans $\operatorname{Ker} t$ ). Dans ces conditions, l'application $t: E \rightarrow R$ vérifie toutes les conditions d'un morphisme stratifié -sans éclatement pour raison de dimension. On se servira alors du Corollaire:

Corollaire 2.C.2. Si $c, c^{\prime}$ sont deux points de la même strate de J, les contre-images $E \cap t^{-1}(c)=C, E \cap t^{-1}\left(c^{\prime}\right)=C^{\prime}$ sont des ensembles stratifiés isotopes (isomorphes).

Application du Théorème 1.G.1.

D. Applications transversales sur un $W$-objet. Soit $A$ un $W$-objet dans $R^{n}$; soit $j: R^{k} \rightarrow R^{n}$ un plongement différentiable propre $(k<n)$. On dira que $j$ est transverse sur $A$ si $j$ est transverse à toutes les strates de $A$. Pour éviter d'avoir à introduire des topologies non métrisables (topologie de Whitney), limitons l'espace source à une boule fermée $D^{k} \subset R^{k}$, telle que l'image par $j \mathrm{du}$ bord $\partial D^{k}$ ne rencontre pas $A$. On vérifie immédiatement que l'ensemble réunion $A \cup_{j}\left(D^{k}\right)$ est un $W$-objet: ses strates sont celles de $A$,-éventuellement partagées par les nouvelles strates de la forme $j\left(D^{k}\right) \cap X$, où $X$ est une strate de $A$, ainsi que l'image $j\left(D^{k}\right)-j\left(D^{k}\right) \cap A$. Les propriétés $A$ et $B$, (Nf), se vérifient sur cet ensemble: en effet, les propriétés $A, B$ pour l'injection $Y \rightarrow \bar{X}$ se conservent par section transversale de $Y$. Par ailleurs, dés qu'une application $j$ est transverse sur une strate $Y$, elle est-en raison de la propriété $A$-transverse sur les strates de l'étoile de $Y$ dans un voisinage de $Y$. Ceci montre que la propriété $j$ transverse à $A$ définit un ouvert dans l'espace $\mathrm{Pl}\left(D^{k}, R^{n}\right)$ des plongements de $D^{k}$ dans $R^{n}$, muni de la $C^{r}$ topologie ( $r$ assez grand); par ailleurs, en raison des théorèmes standard de transversalité, cet ouvert est dense.

ThÉoRł̀me 2.D.1. Dans l'espace $\mathrm{Pl}\left(D, \boldsymbol{R}^{n}\right)$ des plongements du disque $D^{k}$ dans $\boldsymbol{R}^{n}$, l'ensemble des plongements transverses sur un $W$-objet $A$ est dense.

Soit maintenant $j_{t}$ une homotopie de plongements transverses, $t$ variant dans $[0,1]$. Si l'on rajoute une coordonnée $t$ à $R^{n}$ on vérifie que la réunion $j\left(D^{k} \times I\right) \cup A \times I$ est un $W$-objet dans $R^{n+1}$; de plus $t$ est de rang maximum sur chaque strate, et il n'y a pas de sommets intérieurs à $0<t<1$. On peut par suite appliquer le Théorème 2.C.1 et le Corollaire 2.C. 2 ce qui donne:

Les contre-images $j^{-1}(A), j_{1}^{-1}(A)$ sont des ensembles stratifiés isotopes dans $D^{k}$. Nous énoncerons, plus généralement:

ThÉorìme 2.D.2. Si $A$ est un $W$-objet dans $\boldsymbol{R}^{n}$, presque toute appli- 
cation $f: R^{m} \rightarrow R^{n}$ est transverse sur $A$; sif et $g$ sont deux telles applications, assez voisines pour être reliées par une homotopie d'applications transverses, les contre-images $f^{-1}(A), g^{-1}(A)$ sont des $W$-objets stratifiés isomorphes isotopes dans $\boldsymbol{R}^{m}$.

On passe du cas du plongement $j$ au cas d'une application $f$ par l'artifice classique qui consiste à introduire le graphe de $f$ dans $\boldsymbol{R}^{m} \times \boldsymbol{R}^{n}$, graphe qu'on rend transversal sur l'ensemble stratifié $\boldsymbol{R}^{m} \times A$.

REMARQUE. La définition de $W$-objet-qui ne fait intervenir que des propriétés différentielles locales-peut être étendue dans une variété; on vérifiera également que, dans le produit de deux variétés, le produit de deux $W$-objets est un $W$-objet.

\section{Ensembles Analytiques}

\section{A. Ensembles semi-analytiques.}

DÉfinition 3.A.1. Un compact $K$ de $\boldsymbol{R}^{n}$ est dit semi-analytique, si $K$ est réunion finie de compacts $K_{t}$ dont chacun est l'ensemble défini par un système d'inégalités et d'équations:

$F_{i}=0, G_{j} \leqq 0$, où les $F$ et $G$ sont (localement) analytiques. On a le théorème suivant:

THÉORÈme 3.A.1. Tout ensemble semi-analytique compact de $R^{n}$ est un W-objet.

Une démonstration se trouve dans le cours de S. Łojasiewicz [2]. Nous allons rappeler ici, toutefois, les pas essentiels de la démonstration.

Lемме 3.A.2. Soit $Y$ un sous-ensemble analytique (réel ou complexe) de l'ensemble analytique $X$ dans $\boldsymbol{R}^{n}$ (ou $C^{n}$ ); supposons $Y$ et $X-Y$ lisses. $I l$ existe alors un vrai sous-ensemble analytique $Z$ de $Y$, tel que, en tout point de $Y-Z, X$ possède les propriétés (A) et (B) par rapport à $Y$.

Ce lemme fondamental a été établi par H. Whitney [6]; un outil essentiel pour obtenir le résultat est le

Lemme 3.A.3. Lemme de L'Aile (Wing's Lemma). Soit comme plus haut $Y^{r} \subset X^{r+s}$ deux ensembles analytiques locaux (réels ou complexes), $Y$ étant lisse et adhérent d l'ensemble $U$ des points lisses de $X-Y$. Sur le complémentaire d'un vrai sous-ensemble analytique $Z$ de $Y$, tout point y a la propriété suivante: il existe une sous-variêté analytique $W$ de dimension $r+1$, contenant $y$, admettant $Y$ comme sous-ensemble singulier tel que $W-Y$ soit lisse, et qu'on ait: $W-Y \subset U$. $W$ est dite une aile d'axe $Y$. 
Ce lemme peut être établi sur le corps complexe et sur le corps réel (en ce dernier cas, la démonstration est un peu plus délicate; dans le premier cas, il suffit d'utiliser une section linéaire locale de dimension appropriée).

Pour établir 3.A.2 à partir de 3.A.3, on forme au dessus de $R^{n}$ le fibré en grassmanniennes $E$ dont la fibre est la variété des $(r+s)$ plans dans $\boldsymbol{R}^{n}$ (ceci pour établir (A); pour établir (B), il est nécessaire, en plus d' "éclater" la variété $Y$ et de prendre le fibré induit de $E$ sur l'espare éclaté $\hat{Y}$ de $R^{n}$ ). En chacun des cas, la propriété (A) ou (B) s'exprime par le fait que la limite du plan tangent $T_{x}(X), x$ point lisse de $X$, lorsque $x$ tend vers $y \in Y$, doit se trouver dans un certain cycle de Schubert de la grassmannienne fibre. S'il existait un ouvert $V$ de $Y$ où cette propriété n'était pas vérifiée, on pourrait trouver une variété de dimension $(r+1)$ située dans $X$ et contenant $Y$, telle que les positions limites $\lim T_{x}(X)$ soient en dehors de ces cycles de Schubert; en observant qu'une aile (réelle) est presque partout une réunion finie de variétés à bord de classe $C^{1}$, on en déduit une contradiction.

Pour passer de 3.A.2 au Théorème 3.A.1, on utilise le résultat classique:

LEMME 3.A.4. Pour un ensemble analytique réel $A$, il existe un vrai sous-ensemble analytique $A^{\prime}$ tel que $A-A^{\prime}$ ne contient que des points lisses.

La démonstration de 3.A.1 procède alors comme suit: pour l'ensemble $K$ donné, on détermine d'abord les ensembles analytiques minimaux en nombre fini qui contiennent $K$; on extrait de ces ensembles les points lisses contenus dans $K$, qu'on décompose en composantes connexes: on obtient ainsi les strates de dimension maximum de $K$; on opère de même sur le complémentaire, qui est semi-analytique. On réitère alors la procédure, en partitionnant les nouvelles strates par rapport à leur adhérence aux anciennes: on utilise ici le lemme suivant:

LEMME 3.A.5. Si $X, Y$ sont semi-analytiques $X \supset Y$, et si $U$ est une composante connexe de $X-Y$, alors $Y \cap \bar{U}$ est semi-analytique dans $Y$.

Voir [2] pour une démonstration.

En appliquant 3.A.3, on partitionnera ces strates de manière que les strates de dimension maximum possèdent les propriétés (A) (B) par rapport aux nouvelles strates. Comme cette propriété subsiste même si on partitionne ultérieurement ces strates, on pourra itérer la procédure, en diminuant à chaque pas la dimension des strates 
considérées. Ainsi cette proc édure s'achève, et définit $K$ comme un $W$-objet.

Le Théorème 3.A.1 a de nombreuses applications, car il permet de donner un sens à l'expression: l'application différentiable $f: \boldsymbol{R}^{m} \rightarrow \boldsymbol{R}^{\boldsymbol{n}}$ est transversale sur le sous-ensemble semi-analytique $K$. Il suffit pour cela que $f$ soit transverse sur toutes les strates d'une stratification de $K$, par exemple, celle donnée par la construction du théorème 3.A.1, qui est la moins "fine" possible. Lorsque $f$ est transverse sur $K$, et propre, la contre-image $f^{-1}(K)$ est un $W$-objet $A$, et, lorsqu'on déforme $f$ de telle manière que $f$ demeure transverse à $K$, la contreimage $A$ varie en restant isotope à elle-même dans l'espace source (Corollaire 2.C.2).

Corollaire 3.A.6. Soit $A$ un germe d'ensemble analytique réel en $0 \in \boldsymbol{R}^{n}$, et soit $z$ un jet d'ordre $r-1$ de $\boldsymbol{R}^{m}$ dans $\boldsymbol{R}^{n}$; désignons par $M(z)$ la contre-image de $z$ dans l'application restriction $J^{r}(m, n) \rightarrow J^{r-1}(m, n)$; il existe alors un vrai sous-ensemble analytique de $M(z)$, soit $B$, tel que tout jet $\boldsymbol{z}^{\prime} d u$ complémentaire $M(z)-B$ présente la propriété suivante: toute application locale $g$ de jet $z^{\prime}$ est telle que la contre-image $g^{-1}(A)$ est localement stratifiée, et deux applications $g, h$ de même jet $z^{\prime}$ présentent des contre-images $\mathrm{g}^{-1}(A), h^{-1}(A)$ localement isotopes.

Ce résultat généralise un théorème annoncé dans un papier antérieur (Théorème 3 de [5]), et la démonstration est en tout point analogue.

B. Projection des ensembles semi-analytiques compacts. Soit $A$ un ensemble semi-analytique compact de $R^{n+k}, B$ son image par une projection linéaire $p: R^{n+k} \rightarrow R^{n}$. On désignera par la notation PSA (projection d'un semi-analytique) tout sous-ensemble susceptible d'une telle définition.

Lемме 3.B.1. Tout PSA est réunion finie (non disjointe en général) de variêtés analytiques.

On part de la stratification de $A$ comme $W$-objet; à toute strate $X$ de $A$ on associe $S(X)$, l'ensemble critique de la restriction de $p$ à $X$; c'est un ensemble analytique dans $X$ qu'on peut substratifier, et auquel on peut réitèrer la procédure. Finalement on obtient une décomposition de $A$ en variétés analytiques sur lesquelles $p$ est de rang constant. (On peut supposer que cette partition est une stratification.) En vertu du théorème du rang constant, l'image $p(X)$ de toute strate est une variété localement plongée dans l'espace-but; (ces variétés peuvent évidemment se recouper). 
Enonçons le

Lemme 3.B.2. L'intersection de deux PSA est un PSA. Soit $B=p(A)$, où $A$ est semi-analytique dans $R^{n+k}$; et $D=q(C)$, où $C$ est semi-analytique dans $R^{n+k^{\prime}}$ et $q$ la projection linéaire $q: R^{n+k^{\prime}} \rightarrow R^{n}$.

Formons le produit $\boldsymbol{R}^{n+k} \times \boldsymbol{R}^{n+k^{\prime}}$, et désignons par $p_{1}$ la projection sur le premier facteur $\boldsymbol{R}^{n+k}$; désignons dans ce produit par $Y$ la sousvariété linéaire des couples $m, m^{\prime}$ tels que $p(m)=q\left(m^{\prime}\right)$. On vérifie de suite que l'intersection $B \cap D$ est l'image: $B \cap D=$ $p \circ p_{1}\{(A \times C) \cap Y\}$. Comme $A \times C$ est compact, $(A \times C) \cap Y$ est un semi-analytique compact.

DÉfinition 3.B.1. PSA-incidence. Soient $Y, X$ deux variétés analytiques lisses dans $R^{n}$, avec $Y \subset \bar{X}$. On dira que $Y$ est $P S A$ incidente à $X$, s'il existe un $R^{n+k}$ contenant des ensembles semianalytiques $A$ et $B A \supset B$, tels que la partie lisse $P L(B)$ de $B$ soit adhérente à la partie lisse $P L(A-B)$ de $A-B$, et tels que, si $p$ désigne la projection linéaire $p: R^{n+k} \rightarrow R^{n}$, on ait $Y=p(P L(B))$, $X=p(P L(A-B))$ avec $p$ de rang constant sur $P L(B)$ et $P L(A-B)$. Cette définition est bien évidemment de caractère local au voisinage d'un point $y$ de $Y$.

On va démontrer le

ThÉORÈme 3.B.3. Si $Y$ est PSA-incidente d $X$, alors les propriétés (A) et (B) sont vraies de $X$ par rapport d $Y$, d l'exception d'un vrai sousensemble $Z$ PSA de $Y, P S A$-incident d $X$.

Occupons-nous d'abord de la propriété (A); désignons par $G_{a}(A)$ le plan défini par $p^{-1}\left(p\left(T_{a}(A)\right)\right.$; on dira qu'en $a$ on a la propriété $(A p)$ si

$$
\lim _{a b=0} d\left(G_{b}(B), G_{a}(A)\right)=0 .
$$

Cette propriété est la contre-image par $p$ de la propriété (A) dans $\boldsymbol{R}^{n}$; par ailleurs, la propriété (A) dans $\boldsymbol{R}^{n+k}$ entraine la propriété (Ap); car si $U$ et $V$ sont deux plans avec $\operatorname{dim} U<\operatorname{dim} V$, on a $d(U, V)=0$, $V \supset U, p^{-1} p V>p^{-1} p U$.

Il suffit donc de montrer que, si $T$ est le sous-ensemble analytique de $B$ où (A) n'est pas vérifiée, la projection $p(T)$ sur $Y$ n'est pas localement dense. Si, au voisinage d'un point $t \in T$, il existait un voisinage $W$ de $t$ dans $T$ tel que l'image $p(W)$ soit ouverte dans $Y$, on pourrait faire passer par $t$ une aile locale $J$ d'axe $L \subset W$ située dans $P L(A-B)$, telle que la limite des plans $G_{x}(A)$ pour $x \in J$ tendant 
vers $t$ ait un angle $d$ non nul avec $G_{t}(B)$ et telle que $p \mid L$ soit de corang zéro.

Lemme 3.B.4. Si $p: \boldsymbol{R}^{n+k} \rightarrow \boldsymbol{R}^{n}$, et si $J$ est une aile d'axe $L$, alors, si $p$ est de corang zéro sur $L$ et $J-L$, la projection $p(J)$ est un ensemble semianalytique, une aile d'axe $p(L)$.

Ce résultat se démontre par récurrence sur l'entier $k$; à chaque pas, on peut éliminer une des coordonnées $x_{n+i}$ en transformant les équations locales de $J$ en polynomes distingués par rapport à cette coordonnée-par application du théorème de préparation-, et éliminer ensuite cette variable.

Revenant donc à la démonstration de 3.B.3, la projection $p(J)$ de l'aile $J$ donne une aile $J^{\prime}$ dans $X$, dont le voisinage $p(W)=p(L)$ est l'axe. En remarquant qu'une aile est-en presque tout point de son axe-une réunion de variétés à bord de classe $C^{1}$, on en déduit que $\lim d\left(T_{x}(X), T_{y}(Y)\right)=0$, ce qui contredit la propriété $\lim d\left(G_{t}(B)\right.$, $\left.G_{a}(A)\right)>0$ qui définit l'aile $J$.

L'ensemble exceptionnel $T^{\prime}$ dans $Y$ est la projection par $p$ de l'ensemble exceptionnel $T$ dans $B$, dont l'adhérence est semi-analytique. L'ensemble exceptionnel $p(\bar{T})$ est donc un PSA, et l'injection $T^{\prime}=p(T) \rightarrow \bar{X}$ est une PSA-incidence.

La démonstration de la propriété (B) est en tout point analogue (avec, cependant, une différence: on définit d'abord une propriété $B_{p}$ le long de la variété $B^{0}$ par

$$
\lim _{|a b|=0 ; b=k(a)} d\left(p^{-1} p(b a), G_{a}(A)\right)=0
$$

$k$ rétraction normale d'un tube de $B$ sur $B$ ).

Ici cette propriété n'est plus "a priori" une conséquence de la propriété $B^{\prime}$; mais cela est sans importance pour la suite du raisonnement.

Le Théorème 3.B.3 permet d'établir:

THÉoR̀̀me 3.B.4. Tout ensemble PSA (projection d'un semi-analytique compact) est un $W$-objet.

On part de la réunion finie de variétés immergées $\left(X_{j}\right)$ définie au Théorème 3.B.1. On forme toutes leurs intersections (et self-intersections); la famille des variétés $X$ ainsi obtenue forme une partition de l'ensemble $E$ en variétés plongées, si l'on enlève de toute $X_{i}$ son intersection avec toutes les autres strates (et éventuellement, sa selfintersection) qui ne la contiennent pas. Cette partition présente la propriété suivante: si, en un point $y$ de $Y$, il existe un voisinage ad- 
hérent à une autre variété de la partition, soit $X$, alors $\operatorname{dim} Y<\operatorname{dim} X$, et l'incidence locale est soit analytique, soit PSA. En partant d'une strate de dimension maximum $U$, on peut imposer les propriétés (A) et (B) pour les strates du bord, après un éventuel raffinement de la stratification du bord impliquant l'introduction de nouvelles strates, de dimension plus petite, qui présentent l'incidence PSA par rapport à $U$. Or on a le lemme évident par produit suivi de projection:

Lemme 3.B.5. La composée d'une incidence PSA et d'une incidence analytique (dans un ordre quelconque), ou de deux incidences PSA est une incidence PSA.

Par induction décroissante sur la dimension, on pourra donc définir sur $E$ une partition en variétés qui présentent pour leur incidence la PSA-incidence, et par suite les propriétés (A) et (B); $E$ est donc, avec cette partition, un objet de Whitney. On observera que la stratification présente-en plus des propriétés A et B-la propriété du "lemme de l'aile."

C. Stratification des morphismes analytiques. Revenons à la situation d'un ensemble semi-analytique compact $A \subset R^{n+k}$ et soit $p:\left(\boldsymbol{R}^{n+k} \rightarrow \boldsymbol{R}^{n}\right)$ la projection linéaire obtenue en oubliant les dernières coordonnées $u_{n+j}$ de $R^{n+k}$. Avec l'ensemble $A$ on va considérer l'ensemble saturé $p^{-1} p(A)$; cet ensemble n'est pas semi-analytique, maisau besoin en tronquant par des conditions du type $|u| \leqq K$ pour le rendre compact, c'est un PSA. Il existe une stratification de $A$ et de $p^{-1} p(A)$ avec les propriétés suivantes:

(i) Si $X$ est une strate de $A$, variété analytiquement plongée, $p^{-1} p(X)$ est une réunion de strates de $p^{-1} p(A)$.

(ii) La restriction de $p$ à toute strate $X$ de $A$ est de rang constant.

Pour vérifier (i) et (ii) on part de la stratification de $A$ définie par la condition $p \mid X$ de rang constant; on sature ces strates par rapport à $p$, et on prend l'intersection de toutes ces saturées; on enlève de toute strate les intersections avec les strates qui ne la contiennent pas; soit $U$ une strate maximum saturée; elle ne rencontre plus aucune autre strate; on peut alors imposer sur son bord les conditions A et B en introduisant de nouvelles strates qu'on saturera: cette construction n'affecte pas $U$ elle-même, car si $U$ est saturée de $X$, l'injection $X \rightarrow \bar{U}$ est une injection de variété plongée, qui satisfait à (A) et (B). L'itération de cette opération conduit par suite à des strates "saturées" de dimension strictement décroissante, en sorte que la procédure s'achève.

On introduit alors l'espace quotient $R^{n}=R^{n+k} / p$; dans cet espace, 
la projection $p(A)=E$ est munie ipso facto d'une structure de $W$-objet, telle que la contre-image $p^{-1}(Y)$ d'une strate de $E$ soit une réunion de strates de $A$. On se propose de montrer le

ThÉorème 3.C.1. On peut munir, dans ces conditions; les $W$-objets $A$ et $E$ de stratifications telles que la projection $p: A \rightarrow E$ soit un morphisme stratifié.

Dans ce théorème, la structure semi-analytique de $A$ ne joue aucun rôle; le seul point important est que $A$ et $E$ soient des $W$-objets, avec les conditions suivantes:

(a) la restriction de $p$ à toute strate $X$ de $A$ est de rang constant.

(b) l'image réciproque par $p$ de toute strate $Y$ de $E$ est une réunion finie de strates de $A$, dont chacune s'envoie par $p$ sur $Y$ avec rang constant.

On commence par munir l'espace-but $E$ d'une stratification associée à sa structure de $W$-objet: les lambeaux et les tapis sont définis par des voisinages tubulaires des strates, les rétractions $k_{Y^{\prime}}: L_{Y^{\prime}} \rightarrow Y^{\prime}$ par des champs de plans transverses.

Sur l'espace $A$, on commence par la construction suivante: à toute strate $X$, on associe un champ de plans transverses $k_{X}$ du type suivant: $k_{X}(x)=g(x) \oplus h(x)$, où $g$ est un plan "vertical" voisinage normal de $X$ dans sa saturée $p^{-1} p(X)=p^{-1}\left(X^{\prime}\right), X^{\prime}=p(X)$ et $h(x)$ est un plan "horizontal" dont la projection par $p$ est surjective sur $k_{x^{\prime}}\left(X^{\prime}\right), x^{\prime}=p(x)$. Il est clair qu'on peut définir un tel champ $k$ pour chaque strate $X$; mais il sera en général impossible de définir ces champs $k$ de manière cohérente, la condition (Trans) $k_{x}(X) \subset k_{Y X}^{-1} k_{y}(Y)$ ne pouvant être vérifiée: une condition nécessaire pour qu'il en soit ainsi est que l'application $k_{Y X}: \operatorname{Ker} p|X \rightarrow \operatorname{Ker} p| Y$ soit surjective (application "sans éclatement").

Mais si l'on se restreint à la contre-image d'une strate $X^{\prime}$ de $E$, les champs $k_{X}$ commutent à $p$; si $Y<X$ et si $X^{\prime}=p(Y)=p(X)$, alors, dans un lambeau $L_{Y}(X)$ défini par un voisinage tubulaire de $Y$, on définit la rétraction $k_{Y X}$ de $L_{Y}(X)$ sur $Y$ à l'aide du champ $k_{X}$. On a alors $p_{X}=p_{Y} \circ k_{Y X}$. Ceci permet de définir sur toute contre-image $p^{-1}\left(x^{\prime}\right)$ une structure stratifiée, et l'on peut appliquer le Théorème 1.G.2. Ceci montre que la contre-image de toute strate $X^{\prime} \subset E$ est un espace fibré, et le morphisme $p: A \rightarrow E$ une réunion disjointe de fibrations.

Il reste à montrer qu'on peut "recoller" ces fibrations entre elles de manière à satisfaire aux axiomes des ensembles et morphismes stratifiés. La difficulté essentielle consiste à définir les applications $\boldsymbol{k}_{Y X}$ dans $A$, de manière à satisfaire à la condition (Trans), et à la 
condition de commutation $p \circ k_{Y X}=k_{Y^{\prime} X^{\prime}} \circ p$. Donnons dans ce but une définition:

Définition 3.C.1. Deux submersions $f, f^{\prime}$ d'une variété $X$ dans une variété $U$ seront dites "bien voisines" $\bmod y$ si: (a) $f$ et $f^{\prime}$ sont $C^{0}$ voisines; (b) en tout point $x$ de $X$, il existe une sous-variété $Y \subset C$, telles que $f, f^{\prime}$ restreintes à $Y$, soient des submersions dans $U C^{1}$-voisines (c) le champ des variétés locales $Y$ est $C^{1}$ continu: là où deux telles variétés $Y, Y^{\prime}$ sont définies, l'une est une sous-variété de l'autre.

LEммe 3.C.2. Si deux submersions $f$, $f^{\prime}$ sont bien voisines dans un voisinage relativement compact $V$ de $X$, si $\left|f-f^{\prime}\right|$ est assez petit, alors $f$ et $f^{\prime}$ peuvent être déformées l'une dans l'autre par une homotopie $f_{t}$ telle que $f_{t} \mid V$ sont des submersions. Dans ce lemme, la définition de la norme $\left|f-f^{\prime}\right|$ est évidente: $C^{0}$-norme globale et $C^{1}$ norme pour les restrictions aux variétés $Y$ du champ.

Cela étant, on définit les rétractions $k_{Y X}$ de la stratification de $A$ par une triple induction:

(1) Par induction croissante sur la dimension $\operatorname{dim} Y^{\prime}$ de l'image $Y^{\prime}=p(Y)$,

(2) Par dimension croissante dans la contre-image $p^{-1}\left(Y^{\prime}\right)$,

(3) Par cohauteur croissante de la strate $X$ dans le bord de $Y$ (pour n'importe quelle stratification définie sur $A$ compatible avec la structure de $W$-objet donnée).

Cette induction débute donc en stratifiant la contre-image $p^{-1}\left(m^{\prime}\right)$ de tout sommet $m^{\prime}$ de $E$; on se sert dans ce but des champs "verticaux" $g(x)$ auxquels on peut imposer la condition (Trans).

On définira donc par induction des submersions $k_{Y X}: L_{Y}(X) \rightarrow Y$ sur lesquelles on fera, par induction, l'hypothèse que voici: si on désigne par $k_{y}(X)$ le champ transverse à $Y$ qu'on a défini précédemment, il définit une rétraction $\rho_{Y X}: L_{Y}(X) \rightarrow Y$; on admettra que la rétraction construite par induction $k_{Y X}$ est bien voisine de la rétraction $\rho_{Y X}$ définie par les plans $k_{y}(Y)$. Ce bon voisinage est défini par référence à des sous-variétés contre-images par les applications $k^{-1}$ de strates du bord de $X$. Cette hypothèse est bien vérifiée au départ, puisque, dans une contre-image $p^{-1}\left(m^{\prime}\right)$, les $k$ et les $\rho$ sont les mêmes, étant définis par les plans "verticaux" $g_{x}$.

Décrivons maintenant le pas majeur de l'induction; il s'agit de définir l'application $k_{Y X}$, lorsque $Y^{\prime}=p(Y)$ est incidente à $X^{\prime}=p(X)$, et qu'on a déjà défini tous les $k_{Z X}$, lorsque $Y<Z<X$. On commence par considérer l'espace but $E$; là, la stratification est définie par un champ de plans transverses $g_{Y}$, à $Y^{\prime}$, qui définit une rétraction locale $k_{Y^{\prime} X^{\prime}}: L_{Y^{\prime}}\left(X^{\prime}\right) \rightarrow Y^{\prime}$. La contre-image par $p$ de chaque plan $g_{Y^{\prime}}$, coupe 
transversalement la strate $X$ de $A$, et toute strate $Z$ telle que $Y<Z$ $<X$. En effet, l'application $k_{Y^{\prime} Z^{\prime}} \circ p \mid Z \rightarrow Y^{\prime}$ est submersive comme produit de deux submersions, que l'on ait $Y^{\prime}=Z^{\prime}$, ou $Y^{\prime}<Z^{\prime}$. Il en ira par suite de même pour un système de voisinages tubulaires de ces strates; c'est dans chacune de ces contre-images $p^{-1}\left(g_{Y^{\prime}}\right)$ qu'on va travailler; toutes les sections d'un système de tubes associées aux strates $Z$ coupent tous ces plans suivant des variétés à coins isotopes, et le $g$-plan $g_{y}$ transverse à $Y$ induit dans chaque contre-image $p^{-1}\left(g_{y^{\prime}}\right)$ une rétraction $\rho_{Y}\left(y^{\prime}\right): p^{-1}\left(g_{y^{\prime}}\right) \rightarrow p^{-1}\left(y^{\prime}\right) \cap Y$. Pour définir le lambeau $L_{Y}(X)$, on commence par considérer sa projection $L_{Y^{\prime}}\left(X^{\prime}\right)$, qui est défini par la stratification donnée sur $E$. Soit $s$ une fonction (différentiable à coins) sur $X^{\prime}$ définissant le "rayon" de $L_{Y^{\prime}}\left(X^{\prime}\right)$. On considère de même un tapis sur $Y$, de la forme $\operatorname{Inf}\left(h, p\left(\tau_{Y^{\prime}}\right)\right.$; dans une section $p^{-1}\left(g_{y^{\prime}}\right) \cap Y$, le seul tapis à considérer est donné par la fonction $h$, tapis de $Y$, qui contrôle la surmersion $Y \rightarrow Y^{\prime}$. Dans chacune de ces "fibres" $Y \cap p^{-1}\left(y^{\prime}\right)$, on considère un "intérieur" $I_{\epsilon}$ défini par $h>\epsilon$. Le lambeau $L_{Y}(X)$ sera défini comme la réunion $\rho_{y^{\prime}}^{-1}\left(I_{\epsilon}\right) \cap X, s \circ p=\epsilon$, et une partie "effilée sur le bord" définie par une relation de la forme $s=\phi(h)$, où $\phi(0)=0$, soit $\rho_{y^{\prime}}^{-1}(\epsilon>h>a) \cap\{s \circ p<a\} \cap X$ pour $a$ tendant vers zéro.

Dans la contre-image $\rho_{y^{\prime}}^{-1}\left(I_{\epsilon}\right) \cap X$, désignons par $D X$ la réunion des strates $Z$ telles que $Y<Z<X$, et par $C$ un voisinage tubulaire à coins de cette réunion; de plus, on peut définir un épaississement de $C$, isomorphe au produit $C \times I, t$ désignant le paramètre d'épaisseur, $(C, 0)$ étant "extérieur" dans $X$ à $(C, 1)$. Par hypothèse l'application $k_{Y X}$ est déjà définie en dehors de $C$ par la condition (Trans): $k_{Y X}$ $=k_{Y Z} \circ k_{Z X}$. Dans ces conditions, on définira $k_{Y X}$ à l'intérieur de $(C, 1)$ par $k_{Y X}=\rho_{y^{\prime}}$. Puis, on régularise dans la tranche $C \times I$ ainsi: sur $C[0,1 / 2]$, on prend une homotopie de submersions "bien voisines" $k_{u}: C \rightarrow Y$, telle que $k_{0}=k_{Y X}$, et $k_{1}=\rho_{y^{\prime}} \mid C \rightarrow Y$. Sur la tranche $C[1 / 2,1]$ on use d'une homotopie $k_{2}$ reliant $\rho(x, 0)$ à $\rho_{y^{\prime}}(x, 1), x \in C$. Toutes ces opérations n'introduisent que des submersions bien voisines par rapport aux variétés $k_{\mathbf{Z} X}^{-1}(Z)$ (éventuellement prolongées au tube épaissi $C \times I)$. En effet, $k_{X Y} \mid C$ et $\rho_{y^{\prime}} \mid C$ sont bien voisines; en tout point extérieur à $[C, 1]$, on a une identité de la forme $k_{Y X}$ $=k_{Y Z} \circ k_{Z Y}$; or, par l'hypothèse d'induction $k_{Y Z}$ est bien voisine de la rétraction $\rho_{y^{\prime}} \mid Z$; et la rétraction $k_{Z X}: L_{2}(X) \rightarrow Z$ est $C^{1}$ voisine de l'identité sur toute variété transverse aux fibres $k^{-1}(z)$ dans le lambeau $L_{Z}(X)$. Sur la partie effilée du lambeau $L_{Y}(X)$, soit $\rho_{y^{\prime}}^{-1}(h<\epsilon)$, on procède de même, mais en choisissant la fonction $\phi$ assez petite pour que l'écart entre $k$ et $\rho_{y^{\prime}}$ sur $C$ soit assez petit pour que les homotopies $k_{u}$ et $k_{v}$ puissent être construites dans une contre-image par 
$\rho_{y^{\prime}}$ d'un voisinage de la valeur $a$ considérée. Voir Figure 6 pour un exemple simple de cette construction. Ceci achève par suite la démonstration du Théorème 3.C.1.

Remarque sur l'hypothèse de non éclatement. Comme on l'a vu, les applications $k$ définies par la construction précedénte ne sont pas associées à un système de plans transverses; par suite, elles ne permettent pas de construire une représentation sectorielle de $A$. S'il n'y a pas éclatement $\left(k_{Y X}: \operatorname{ker} p|X \rightarrow \operatorname{ker} p| Y\right.$ surjective) la construction précédente se simplifie: en effet, sur l'ensemble $C$ considéré, on a identiquement $k=\rho_{Y^{\prime}}$, parce qu'on peut construire un champ de plans transverses aux strates de $X$ satisfaisant à la condition (Trans), et se projetant sur un champ de plans transverses $k_{Y}$, aux strates de $E$.

Dans un article antérieur [4], j'ai donné pour exprimer la condition de non-éclatement le fait que, pour tout couple de strates $Y, X$ avec

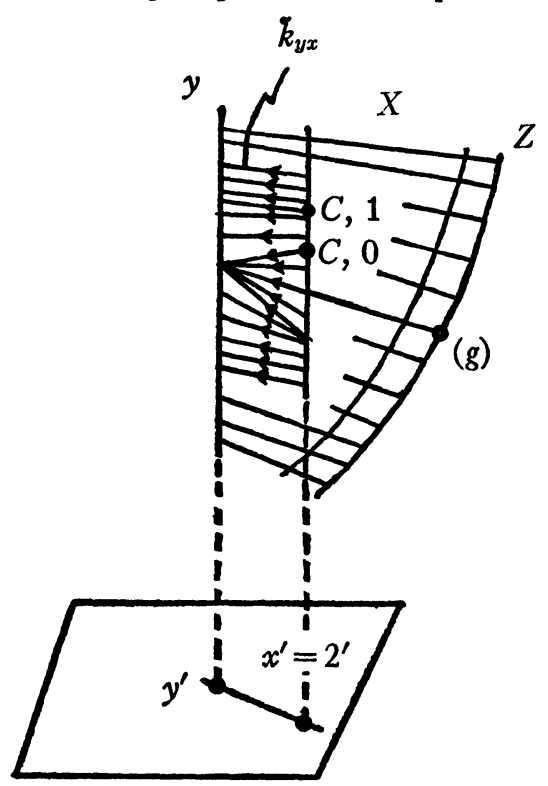

LEGENDE DE LA FIGURE 6

On considère une stratification de $\boldsymbol{R}^{3}\left(O_{x y z}\right)$ ainsi définie: une surface réglée (hélicoide) $(Z)$ contenant l'axe $O_{z}$ qu'on prend pour strate $Y$ de dimension un. $(X)$ est une strate de dimension trois constituée du complémentaire de $R^{3}-Z$ (ou une de ses composantes connexes). On projette cette stratification sur $O x y$ par la projection $p(x, y, z) \rightarrow(x, y) . Y^{\prime}=p(Y)$ est l'origine, $X^{\prime}=p(Z)=p(X)$ le plan $O x y-0$. On a représenté par des lignes flêchées les trajectoires de la rétraction $\tilde{k}_{Y X}$, telle qu'elle est donnée par la construction du Théorème 3.C.1. On n'a représenté ces trajectoires que dans un plan de la forme $y / x=c s t e$, contenant la génératrice $(g)$. Toutes les autres trajectoires se déduisent de celles-là par une transformation hélicoidale laissant $Z$ invariante. 
$Y<X$, le corang de $p$ sur $Y$ est inférieur au corang de $p$ sur $X$. Cette condition nécessaire n'est pas suffisante pour assurer la condition de non-éclatement $k: \operatorname{ker}_{Y X} p|X \rightarrow \operatorname{ker} p| Y$ surjective.

Néanmoins, lorsque $A$ est semi-analytique, on peut montrer que, si $Y$, lisse, est adhérent aux points lisses de $X, X, Y$ analytiques plongées dans $\boldsymbol{R}^{n+k}$, la condition corang $p \mid Y<$ corang $p \mid X$ entraine qu'on a presque partout en $Y$ une sorte de propriété (A): $\lim _{X Y=0} d\left(\operatorname{ker} p: \mid T_{y}(Y)\right.$, ker $\left.p \mid T_{x}(X)\right)=0 d$ étant pris dans le $\boldsymbol{R}^{K}=\operatorname{Ker} p$. (Ceci se démontrerait par une application du lemme de l'aile aux ensembles de variétés linéaires localement contenus dans $X$ resp. $Y$.) Ainsi, après un raffinement éventuel de la stratification, on pourra obtenir la condition de non-éclatement. 11 resterait évidemment à savoir si la propriété reste vraie pour les strates exceptionnelles ainsi introduites.

\section{Théorie des Singularités D'APplications DiffÉRENTIABLES}

On va décrire brièvement ici, comment la théorie des ensembles et morphismes stratifiés peut s'appliquer à la théorie locale des applications différentiables. Nous énoncerons ici, sans démonstration, un certain nombre de lemmes de caractère algébrique sur des variétés algébriques de l'espace des jets $J^{r}(n, p)$ des germes d'applications de $\boldsymbol{R}^{n}$ dans $\boldsymbol{R}^{p}$.

\section{A. Variêtés de l'espace des jets.}

Lemme 4.A.1. Soit $V$ une variété plcngée de $J^{r}(n, p)$, dont la projection canonıque dans $J^{1}(n, p)$ n'est pas contenue dans le jet nul. On suppose codim $V<n$; il existe alors dans la contre-image $p^{-1}(V) \subset J^{r+1}(n, p)$ une vraue sous-variété $s V$ telle que tout jet $z$ du complémentaire $p^{-1}(V)$ - $s V$ a la propriété suivante: tout germe d'application $f$ de jet $z$ à l'orngine est tel que la dérivée $j r(f)$ est transverse d $V$ au point $z$, et que f restreint d la contre-ımage $j^{r}(f)^{-1}(V)$ est une immersion locale dans le but $R^{p}$.

Une variété telle que $p^{-1}(V)-s V$ dans $J^{r+1}$ sera dite $s$-stabilisée: l'opération $s$, appliquée à une variété $s$-stabilisée donne la variété vide.

Si $V$ est une variété algébrique (resp. analytique), il en va de même pour $s V$.

La démonstration du Lemme 4.A.1 présente un caractère purement algibrique [7].

LEMME 4.A.2. Soit $X_{1} X_{2} \cdots X_{8}$ un système de variêtés algébriques localement stratifié (W-objet) au voisinage d'un jet $z \in J^{r}(n, p)$; sup- 
posons toutes ces variétés s-stabilisées au voisinage de $z$; il existe alors dans la contre-image de $z$ dans $J^{r+s}$ un vrai sous-ensemble algébrique $w \subset p^{-1}(z)$, tel que tout jet $z^{\prime} d u$ complémentaire $p^{-1}(z)-w$ a la propriété suivante; tout germe d'application $f$ de jet $\boldsymbol{z}^{\prime}$ est tel que les images par $f$ des variêtés contre-images $j^{r}(f)^{-1}\left(X_{i}\right)$ sont des variétés immergées dans $\boldsymbol{R}^{p}$ (au voisinage de 0 ) qui vérifient pour leurs incidences les propriétés (A) et (B) de Whitney.

Un lemme accessoire dans la démonstration de 4.A.2 est le suivant:

Lemme 4.A.3. Soit $A$ un germe d'ensemble analytique dans $R^{n}$, de dimension $k, \bar{A}$ l'espace des $k$-plans tangents a $A$, considéré comme l'adhérence de la section tangente du fibré en grassmanniennes au dessus des points lisses $A^{0}$ de $A$; sif désigne un germe de plongement analytique $f: \boldsymbol{R}^{m} \rightarrow \boldsymbol{R}^{n}$, transverse sur $A^{0}$, alors la limite pour $x \in f\left(\boldsymbol{R}^{m}\right) \cap A$ tendant vers 0 du plan tangent $T_{x}(A)$ constitue un ensemble semi-algébrique $C$ de la grassmannienne, et cet ensemble ne dépend que du jet (d un ordre assez élevé) $d u$ plongement $f$.

En effet, les propriétés (A) et (B) pour l'incidence de $Y$ sur $X$ font intervenir essentiellement l'ensemble limites des plans tangents à $X$ lorsqu'on tend vers $Y$, donc les ensembles du type $\mathrm{C}$ du lemme précédent. (En fait, il faut considérer dans $\boldsymbol{R}^{n} \times \boldsymbol{R}^{p}$, non seulement les plans tangents aux variétés, mais aussi leurs projections ou-ce qui revient au même-leurs saturés pour la projection $R^{n} \times R^{p} \rightarrow R^{p}$.)

Lемме 4.A.4. Soient $X_{i}$ un système de variétés algébriques localement stratifié en un point $z \in J^{r}(n, p)$, s-et w-stabilisées; dans la contre-image $p^{-1}(z), J^{r+s}(n, p)$, il existe un vrai sous-ensemble algébrique $q$, tel que tout germe f dont le jet est dans $p^{-1}(z)-q$ présente la propriété suivante: les intersections $\cap f \mid\left(j^{r} f\right)^{-1}\left(X_{i}\right)$ et self-intersections des images $f \mid\left(j^{r} f\right)^{-1}\left(X_{i}\right)$ sont en position générale et définissent dans le but un $W$-objet localement stratifié $P$; dans l'espace source, les variêtés $j f^{-1}\left(X_{i}\right) \cap f^{-1}(P)$ forment également un $W$-objet.

Dans ce lemme, on définit le saturé de l'ensemble $X_{i}$ en formant le produit de deux ensembles-sources $S_{1} S_{2}$ qu'on envoie dans le produit du but $B$ par l'espace $J^{r}$ des jets selon l'application: $\left(x_{1}, x_{2}\right) \underset{\rightarrow}{\rightarrow} f\left(x_{1}\right)$ $-f\left(x_{2}\right), j r f_{1}\left(x_{1}\right)$. Le saturé de $X_{i}$ est l'image par la projection $p_{1} S_{1} \times S_{2}$ $\rightarrow S_{1}$ de la contre-image $F^{-1}\left(0, X_{i}\right)$. En vertu du théorème 3.A.6 appliqué à $F$, on peut stabiliser le saturé de $X_{i}$; par le Lemme 4.A.3 on peut également stabiliser l'ensemble $C$ limite des plans tangents le long de toute pré-image d'une intersection $f\left(X_{i}\right) \cap f\left(X_{j}\right)$; ceci suffit par suite à stabiliser leur projection dans le but. Le fait que l'ensemble image est un $W$-objet résulte du fait que, si $f$ est un polynome, 
cet ensemble est semi-algébrique (théorème de Tarski-Seidenberg), donc un $W$-objet. Il suffit donc de stabiliser chacune de ses strates du point de vue de la limite des plans tangents.

\section{B. Stratification canonique de l'espace des jets.}

DÉFInITION 4.B.1. On appelle stratification canonique de l'espace des jets $J(n, p)$ le plus petit système de variétés algébriques stratifié qui contient la stratification canonique de $J^{1}(n, p)$ (celle définie par le rang), et clos par rapport aux opérations $s, w$ et $q$.

Comme les opérations $s, w$ et $q$ accroîssent strictement la codimension, il existe, pour tout couple $n, p$ un ordre $r$ tel que toutes les strates de la stratification canonique de codimension $\leqq n$ sont de ce fait, $s$, w et $q$-stabilisées (i.e., ces opérations définissent l'ensemble vide sur le système des strates canoniques incidentes en ce point). Ceci motive la définition:

DÉFINITION 4.B.2. Un jet $z \in J^{r}(n, p)$ est dit déterminant si la strate canonique qui le contient est $s-w$ et $q$-stabilisée par rapport à toutes les strates de l'étoile dans la stratification canonique.

ThÉORÈme 4.B.1. Si un jet $z \in J^{r}(n, p)$ est déterminant, tout germe $f$ de jet $f$ présente les propriétés suivantes: l'ensemble critique $S(f)$ et son image $f[S(f)]$ sont localement des $W$-objets; l'image par $f$ d'une strate de $S(f)$ est une strate de $f[S(f)]$ de même dimension; $f$ est localement un morphisme stratifié sans éclatement pour la stratification de la source et $d u$ but contenant $S(f)$ et $f[S(f)]$ respectivement.

De plus si $g$ est un autre germe de même jet $z, g$ a localement même type topologique que $f$. De même, si $z$ et $\boldsymbol{z}^{\prime}$ sont deux jets déterminants de la même strate canonique, deux germes de jets $z, z^{\prime}$ ont même type topologique local.

Les propriétés énoncées pour $S(f)$ et $f[S(f)]$ résultent immédiatement des définitions des $s$, $w$ et $q$ stabilisations; on est donc localement dans la situation du Théorème 3.C.1. Le fait que ce morphisme est sans éclatement résulte de la continuité du champ "vertical" ker $f$ : sur une strate formée de points réguliers, $\operatorname{dim} \operatorname{ker} f=n-p$, et il y a continuité de ce champ; sur une strate de points critiques ker $f$ est réduit à zéro. L'affirmation sur les types topologiques locaux de $f$ et $g$ résulte alors du Théorème 1.G.2.

Définition 4.B.3. Un jet déterminant $z$ est dit stable, si la codimension de la strate canonique le contenant est inférieure à $n$. Il est clair que si $f$ présente $z$ en 0 , toute $C^{m}$ déformation $g$ de $f$ présente un jet de la même strate canonique en un point voisin (en fait une sous-variété de codimension égale à la codimension de cette strate). Ceci résulte immédiatement du lemme de transversalité. Il en résulte également le 
Corollaire 4.B.2. Tcute application $f$ d'une variêté $X$ dans $Y$ peut être approchée par une application g qui ne présente que des jets stables, et cette propriêté est ouverte pour la $C^{m}$ topologie, $m$ assez grand.

C. Déploiement universel d'un jet déterminant. Lorsqu'un jet déterminant $z$ appartient à une strate canonique de codimension $k$ dans $J^{r}(n, p)$, on peut définir une famille à $k$ paramètres de germes dont la dérivée $j^{r}$ est transverse sur cette strate. Une telle famille est bien définie à un isomorphisme stratifié près; elle paramétrise évidemment tous les types topologiques locaux de germes voisins du germe donné $f$ de jet $\boldsymbol{z}$. Comme la stratification canonique est localement finie, cette famille ne comporte qu'un nombre fini de types topologiques voisins du type donné.

C'est cette famille-associée à un jet stable de $\boldsymbol{R}^{n+k}$ dans $\boldsymbol{R}^{p+k}$ qu'on appelle le déplonement universel de jet $z$. Ceci montre que la stratification canonique stabilisée de $J(n, p)$ s'envoie isomorphiquement-par suspension-dans la stratification canonique stabilisée de $J(n+k, p+k)$.

Lorsque le jet d'un germe $f$ est déterminant, tous les jets de $f$ voisins de 0 sont stables. Il semble probable qu'un jet non déterminant admet toujours une infinité de réalisations ayant des types topologiques différents. En effet, dans tous les exemples connus, un jet non déterminant admet une réalisation dans laquelle, ou l'une des strates inverses des strates canoniques, ou l'une de leurs images, ou l'une des intersections des images dans le but, a une dimension excédentaire, conformément à la maxime philosophique générale selon laquelle toute instabilité topologique est due à un manque de transversalité.

\section{REFERENCES}

1. W. Browder, J. Levine, G. Livesay, Finding a boundary of an open manifold, Amer. J. Math. 87 (1965), 1017-1028.

2. S. Lojasiewicz, Ensembles semi-analytiques, Cours Faculté des Sciences d'Orsay, Mimeographié I.H.E.S., Bures-sur-Yvette, July, 1965.

3. J. Mather, Stability of $C^{\infty}$ mappings. I, Ann. of Math. (2) 87 (1968), 89-104; II (to appear).

4. R. Thom, La stabilite topologique des applications polynomiales, Enseignement Math. 8 (1962), 24-33.

5. - Local topological properties of differentiable mappings, Colloquium on Differential Analysis, Tata Institute, Bombay, 1964, Oxford Univ. Press, London, 1964, pp. 191-202.

6. H. Whitney, Local properties of analytic varieties, M. Morse Jubilee Volume, Differential and Combinatorial Topology, Princeton Univ. Press, Princeton, N.J., 1965, pp. 205-244.

7. J. M. Boardman, Singularities of differentiable maps, Inst. Hautes Etudes Sci. Publ. Math. 33 (1967), 383-419. 